\title{
Morphofunctional changes at the active zone during synaptic vesicle exocytosis
}

This manuscript (permalink)was automatically generated from aseedb/synaptic tomo ms@cfed8af on March 5, 2022.

\section{Authors}

\section{- Julika Radecke*}

\section{(D) 0000-0002-5815-5537 · () julikaradecke}

Institute of Anatomy, University of Bern, Bern, Switzerland; Department of Neuroscience, Faculty of Health and Medical Sciences, 2200 Copenhagen N, University of Copenhagen, Copenhagen, Denmark; Diamond Light Source Ltd, Didcot, Oxfordshire, United Kingdom · Funded by Swiss National Science Foundation, P2BEP3_172242

\section{- Raphaela Seeger ${ }^{*}$}

- P elatella

Institute of Anatomy, University of Bern, Bern, Switzerland; Graduate School for Cellular and Biomedical Sciences,

University of Bern

\section{- Anna Kádková}

\section{(iD) 0000-0001-6648-9679}

Department of Neuroscience, University of Copenhagen, Blegdamsvej 3B, 2200 Copenhagen N, Denmark

- Ulrike Laugks

\section{(D) 0000-0003-4175-4354}

Max-Planck-Institute of Biochemistry, Am Klopferspitz 18, 82152 Martinsried, Germany

\section{- Amin Khosrozadeh}

\section{- () ameen-khosrowzadeh}

Institute of Anatomy, University of Bern, Bern, Switzerland; Graduate School for Cellular and Biomedical Sciences, University of Bern

\section{- Kenneth N. Goldie}

\section{(iD) 0000-0002-7405-0049}

BioEM Lab, Biozentrum, University of Basel, Basel, Switzerland

- Vladan Lučić

\section{(iD) 0000-0003-3698-7436 · vladanl}

Max-Planck-Institute of Biochemistry, Am Klopferspitz 18, 82152 Martinsried, Germany

\section{- Jakob B. Sørensen}

\section{(iD) 0000-0001-5465-3769 $\cdot$ (D) J BSorensen}

Department of Neuroscience, University of Copenhagen, Blegdamsvej 3B, 2200 Copenhagen N, Denmark · Funded by Novo Nordisk Fonden, NNF17OC0028516.; Carlsbergfondet, CF17-0875; Independent Research Fond Denmark, 802000228A; Lundbeckfonden, R277-2018-802

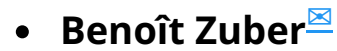

\section{(iD) 0000-0001-7725-5579 • (?) aseedb}

Institute of Anatomy, University of Bern, Bern, Switzerland · Funded by Swiss National Science Foundation, 179520; ERA-NET NEURON, NEURON-119 
bioRxiv preprint doi: https://doi.org/10.1101/2022.03.07.483217; this version posted March 7.2022. The copyright holder for this preprint (which was not certified by peer review) is the author/funder, who has granted bioRxiv a license to display the preprint in perpetuity. It is made available under aCC-BY 4.0 International license.

凶Address correspondence to benoit.zuber@ana.unibe.ch and jakobbs@sund.ku.dk.

\section{* These authors contributed equally.}




\section{Abstract}

The fusion of synaptic vesicles (SVs) with the plasma membrane (PM) proceeds through intermediate steps that remain poorly resolved. Additionally, the effect of persistent high or low exocytosis activity on intermediate steps remains unknown. Through time-resolved cryo-electron tomography, we ordered events into a sequence. Following stimulation, proximal tethered SVs rapidly form additional tethers with the PM. Simultaneously, fusion initiation occurs by membrane curvature ('buckling') of the SV and PM. It is followed by the formation of a fusion pore, and the collapse of SV membrane. At this time, membrane-proximal, but not membrane-distal, vesicles lose their interconnections, allowing them to move towards the PM. Two mutations of SNAP-25 that arrests or disinhibit spontaneous release, respectively, both caused a loss of interconnectors, while the disinhibiting mutant also caused a loss of membrane proximal multiple-tethered SVs. Overall, tether formation and connector dissolution is triggered by stimulation and respond to the spontaneous fusion rate. These morphological observations likely correspond to the transition of SVs from one functional pool to another.

\section{Introduction}

For normal brain function such as movement coordination or memory formation, communication between neurons is essential. In the central nervous system, neurons communicate through the release of neurotransmitters at synapses. This process relies on synaptic vesicle (SV) exocytosis, i.e. the fusion of neurotransmitter-filled SVs with the plasma membrane (PM). SV exocytosis involves a sequence of steps [1,2]. The vesicle is docked to the active zone (AZ) PM and the exocytosis machinery goes through a maturation process, termed priming, after which the SV is ready to fuse. These SVS form the readily releasable pool (RRP). Finally, a calcium influx triggers fusion of the SV with the PM. Docked SVs are defined as those in very close proximity or direct contact with the PM as observed by electron microscopy (EM), whereas priming refers to SV ability to undergo exocytosis immediately upon stimulation. Whether every docked SV is also primed has been debated $[1,3,4]$. A high-pressure freezing/freeze-substitution EM study of synapses has indicated that vesicles which are in direct contact with the PM, i.e. docked, are also primed and belong to the RRP and that this situation occurs downstream of vesicle tethering [4]. From a molecular perspective, priming involves several proteins, including the SNARE complex (SNAP-25, syntaxin-1, and synaptobrevin-2), Munc13, Munc18, synaptotagmin-1, and complexin [2,5]. All three SNAREs form a highly stable tight four-helix bundle, known as trans-SNARE complex. The surfaces of the SV and the PM, are both negatively charged and therefore tend to repulse each other. The formation of the trans-SNARE complex counteracts this repulsion and brings the SV and the PM in high proximity [6]. Evidence has suggested that the SNARE complex is only partially zipped in primed vesicles [7]. Furthermore, various studies have suggested that the formation of at least three SNARE complexes provides the necessary energy for a vesicle to become fusion-competent $[\underline{8}, 9,10]$. Yet in the absence of cytoplasmic $\mathrm{Ca}^{2+}$, minimal spontaneous exocytosis takes place. When the presynaptic terminal gets depolarized by an action potential, $\mathrm{Ca}^{2+}$ flows in the cytoplasm and binds to synaptotagmin-1, which is localized at the SV surface. Upon $\mathrm{Ca}^{2+}$ binding, synaptotagmin-1 was proposed to insert between the head groups of the PM anionic phospholipids and trigger membrane curvature and destabilization, leading first to hemifusion and subsequently to fusion [11]. Interestingly, much of the trans-SNARE bundle surface is negatively charged. This contributes to the electrostatic barrier that minimizes spontaneous fusion. Synaptotagmin-1 can then act as an electrostatic switch that triggers exocytosis. Introducing negatively charged side chains by site-directed mutagenesis reduces the rate of spontaneous and evoked exocytosis, whereas introducing more positive side chains enhances the rate of spontaneous exocytosis and depletes the RRP. [12] 

made available under aCC-BY 4.0 International license.

Cryo-electron tomography (cryo-ET), which preserves samples to atomic resolution, revealed that under resting conditions, no SV is in direct contact with the PM and the majority of AZ-proximal SVs are connected to the PM by a variable number of short tethers $[13,14]$. The observed gap between the SV and the PM is consistent with the model of an electrostatic barrier formed by the negative charges of the SV, the PM, and the trans-SNARE bundle [12]. In synaptosomes treated with hypertonic-sucrose solution, which depletes the RRP, the majority of tethered vesicles had only 1 or 2 tethers $[13,15,16]$. This observation suggested that the RRP consists of SV, which are linked to the PM by 3 or more tethers. The RRP, as identified by morphological criteria, only represents a minority of AZ-proximal vesicles. This is in agreement with previous reports. In one of them, the term pre-primed pool was used for the few vesicles ( $\sim 1$ vesicle at hippocampal synapses) that are rapidly released and another publication showed that the RRP is made up of only 10-20\% of SVs located on the AZ (equal to $\sim 1$ vesicle on hippocampal synapses) $[17,18]$. The ensemble of proximal vesicles that are not in the RRP have been termed non-RRP and presumably belong to the recycling pool that releases more slowly $[13,19]$. Farther away from the AZ, partially intermixed with the recycling pool, is the reserve pool containing vesicles that only release upon high frequency stimulation. Vesicles in the reserve pool are tightly clustered and well inter-connected by structures that were termed connectors $[13,19]$. It should be noted, that the molecular nature of connectors is not known and is possibly heterogenous. Synapsin has been proposed as a molecular constituent. However, since the deletion of all forms of synapsin does not lead to the complete absence of connectors, it is clear that not all connectors contain synapsin $[20,21]$. The second row of SVs near the active zone (45-75 nm from AZ), immediately after the proximal vesicles ( $<45 \mathrm{~nm}$ from AZ), is called the intermediate region. Resting state intermediate SVs are less densely packed and also less connected than proximal SVs [14]. This suggests that, after exocytosis of RRP SVs, intermediate SVs could be rapidly recruited in the RRP by diffusion [22]. Synaptic activity enhances the mobility of a fraction of SVs, whereas it induces synapsin dissociation from SVs in a synapsin phosphorylation-dependent manner [23,24]. The same mobility enhancement can be achieved through inhibition of synapsin dephosphorylation, which leads to synapsin dissociation from SVs, or by knocking out all three synapsin forms $[25,26,27]$. Interestingly, ribbon synapses do not express synapsin and show higher SV mobility than conventional synapses [28]. It is therefore conceivable that inter-SV connectors restrain SV diffusion and that synaptic activity influences the level of inter-SV connectivity and thereby their mobility.

To investigate this hypothesis and to better understand the impact of depolarization and synaptic activity on SV tethering, we designed two sets of cryo-ET experiments. On the one hand, we compared the morphology of wild-type rat synaptosomes in resting state and a few milliseconds after depolarization. On the other hand, to study the consequences of increased or decreased spontaneous synaptic activity, we imaged synapses in mouse neuronal culture expressing either wild-type SNAP-25, a more positively charged SNAP-25 mutant (4K mutant), or a more negatively charged mutant of SNAP-25 (4E mutant) [12] The more positively charged SNAP-25 mutant, which is constitutively active, showed no triple-tethered SV [12]. This confirmed the morphological definition of the RRP. Our experiments revealed an immediate formation of additional tethers between proximal RRP vesicles and the PM after depolarization. Shortly after exocytosis the level of inter-SV connectivity was decreased among SVs situated in a 25 to 75-nm distance range from the AZ PM. Altogether, our results indicate a regulation through connectors of SV mobility and their recruitment at the AZ PM.

\section{Results}

To analyze the morphological changes occurring in the presynpase shortly after stimulation, we pursued a time-resolved cryo-electron tomography approach. A 52-mM KCl containing buffer was sprayed with an atomizer to depolarize synaptosomes and stimulate exocytosis milliseconds before vitrification. The spray droplet size was optimized by cutting a 1-ml pipet tip to a diameter matching an EM grid ( $3 \mathrm{~mm}$ ) and fixed to the atomizer glass outlet to disperse the spray (Figure $1 \mathrm{~A} 1$ ).

Furthermore, to achieve the shortest possible delay between spraying and freezing, the nozzle was set 1-2 $\mathrm{mm}$ above the liquid ethane container. This generated many small spray droplets spread 
bioRxiv preprint doi: https://doi.org/10.1101/2022.03.07.483217; this version posted March 7,2022 . The copyright holder for this preprint (which was not certified by peer review) is the author/funder, who has granted bioRxiv a license to display the preprint in perpetuity. It is made available under aCC-BY 4.0 International license.

throughout the grid (Figure 1 A2-A4). Even if sprayed droplets were well distributed throughout the grid, not all synaptosomes were in contact with exocytosis-triggering $\mathrm{KCl}$ solution. Given the very low throughput of cryo-electron tomography, we followed a correlative light and electron microscopy approach. By cryo-fluorescence microscopy, we identified areas where synaptosomes fluorescently labeled by calcein blue and spray droplets labeled by fluorescine were colocalized. Additionally, phase contrast imaging enabled quality control of the frozen EM grid with respect to ice contamination and ice cracks, as shown previously [29]. 9 control and 9 stimulated synaptosome tomograms were analyzed (Supplementary Figure \{fig:suppl_tomogram_slices\}A and B, Supplementary Movie S1). We restricted our analysis to synaptosomes that possessed a smooth $\mathrm{PM}$, free of signs of rupturing and that had a mitochondrion, as we considered these factors essential for synaptosome function.

In addition, we manipulated the electrostatic state of the SNARE complex through mutated SNAP-25 protein introduced using lentiviral vectors into primary SNAP-25 knockout neurons grown on EM grids [12] (Figure 1B1-B4). The "4E" mutation contains four glutamic acid substitutions, whereas the " $4 \mathrm{~K}$ " contains four lysine substitutions; all mutations are placed in the second SNARE-motif of SNAP-25 and were shown to decrease and increase the rate of spontaneous miniature release, respectively [12]. Optimization of Primary neurons culturing conditions allowed us to establish a protocol, which provides functional synapses thin enough for direct imaging by cryo-ET. Astrocytes were added to 12 well plates and were grown for 2 days. After 2 days, the medium was exchanged to a medium that favors neuronal growth and impedes astrocyte growth. At the same time a droplet of the neuronal suspension was added onto a flame sterilized EM grid and incubated for $30 \mathrm{~min}$ at $37^{\circ} \mathrm{C}$, hereafter the grids were placed into the 12 well plates containing the astrocytes. Neurons were grown for 10-14 days until plunge freezing and were then analyzed at a Titan Krios by cryo-ET (Supplementary Figure \{fig:suppl_tomogram_slices\}C and D, Supplementary Movie S2. Thereby, we could image chronically overactive or inactive synapses and relate presynaptic architectural modifications to different functional states.
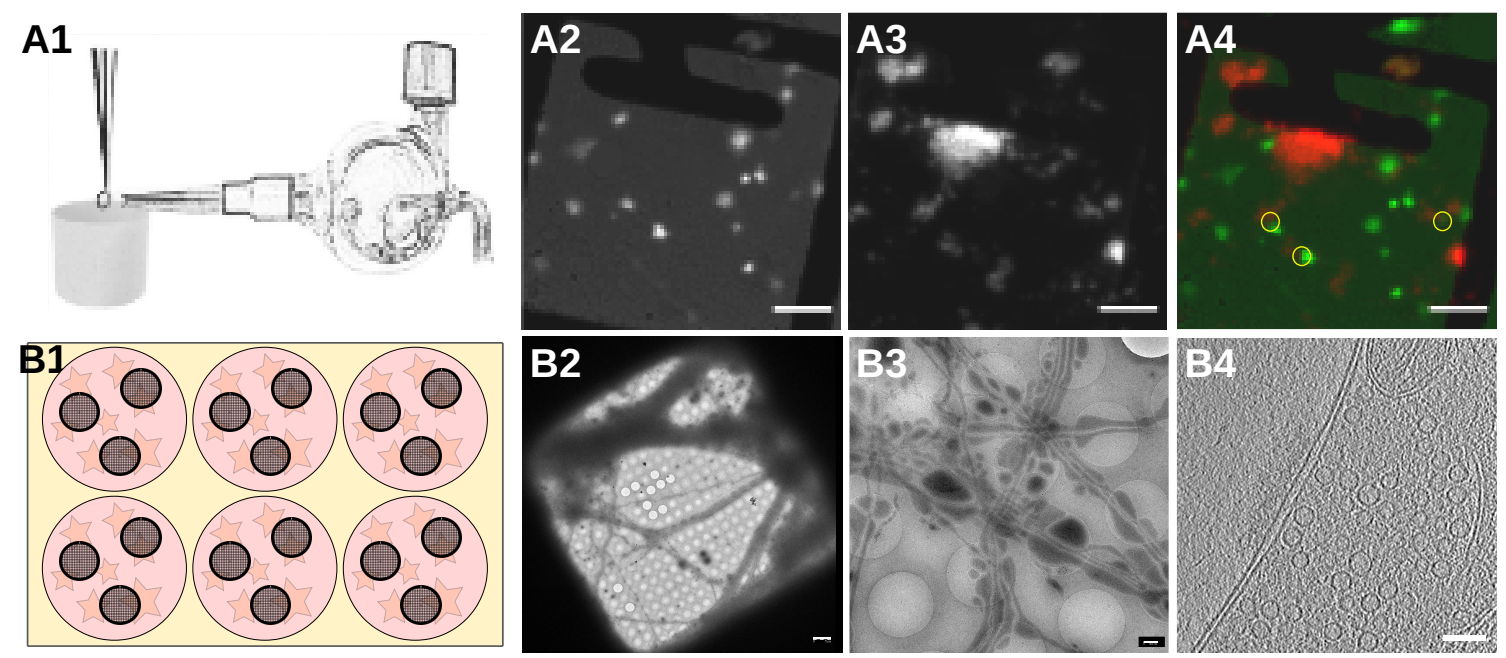

Figure 1: Experimental models. A1) Glass atomizer used to disperse depolarizing solution on the EM grid milliseconds before the grid is vitrified. A2) Spray droplets imaged with the GFP filter set. A3) Synaptosomes imaged with the DAPI filter set. A4) Overlay of spray droplets (green) and synaptosomes (red). Scale bars, 20 um. B1) Schematic drawing of a 6well petridish depicting astrocytes (pink stars) growing at the bottom of the petridish and EM grids (black round grid overlaying the astrocytes) with neurons growing on the grids. B2) Gridsquare overview with neurons growing over it; scale bar $=5 \mu \mathrm{m}$. B3) Medium magnification overview of neurons growing over R2/1 holes; scale bar = 500 $\mathrm{nm}$. B4) One slice of a tomogram depicting the synapse and its respective postsynapse; scale bar $=100 \mathrm{~nm}$.

\section{Increased membrane curvature at the onset of exocytosis}

We analyzed the morphology of SVs fusing with the AZ PM. Synaptosomes of a single grid have not all been stimulated for the same duration. Some synaptosomes have been in contact with the stimulating solution from the moment the droplet touched the grid, while others were not at all in contact with the stimulating solution because no droplet landed close enough to them. Importantly, 
bioRxiv preprint doi: https://doi.org/10.1101/2022.03.07.483217; this version posted March 7,2022 . The copyright holder for this preprint (which was not certified by peer review) is the author/funder, who has granted bioRxiv a license to display the preprint in perpetuity. It is made available under aCC-BY 4.0 International license.

some synaptosomes were located near the point of impact of a droplet, in which case, it took some time for $\mathrm{KCl}$ to diffuse until the concentration around these synaptosomes rose sufficiently to trigger exocytosis. Therefore, the time interval between triggering exocytosis and freezing ranged between 0 ms and the interval between spray droplets hitting the grid and freezing, which was comprised between 7 and 35 ms depending on the experiments (see [30]). This time window, and the diffusional delay of the $52 \mathrm{mM} \mathrm{KCl}$, made it possible to identify synaptosomes at different stages of fusion.

Synaptosomes from both control and sprayed grids were thoroughly analyzed for signs of exocytosis, which consisted of morphological changes of the AZ PM and the tethered SV occurring upon stimulation, which are described hereafter. These signs were only detected in synaptosomes from sprayed grids and are presented in the most parsimonious chronological order. Upon stimulation, both the vesicle membrane and the PM were slightly bent towards each other (Figure 2B1-B3; orange arrows). These structures, which have previously been reported in liposomes but not in synapses, have been referred to as membrane curvature events [11]. Control synaptosomes (i.e. not sprayed) on the other hand, had a straight PM, and no SV membrane was buckled (Figure 2A). Following membrane bending, we observed contacts between vesicles and the PM bilayer where both membranes lose their clear contours (Figure 2C1 \& C2; pink arrows). This was followed by further transitioning states prior to and during pore opening (Figure 2D-F; blue arrows). In the next observed fusion state, the vesicle was wide open (Figure $2 \mathrm{G}$ ), followed by almost completely collapsed vesicles where only a small bump on the PM remained visible (Figure $2 \mathrm{H}$ ). These structures were not observed in any of the non-sprayed control datasets.

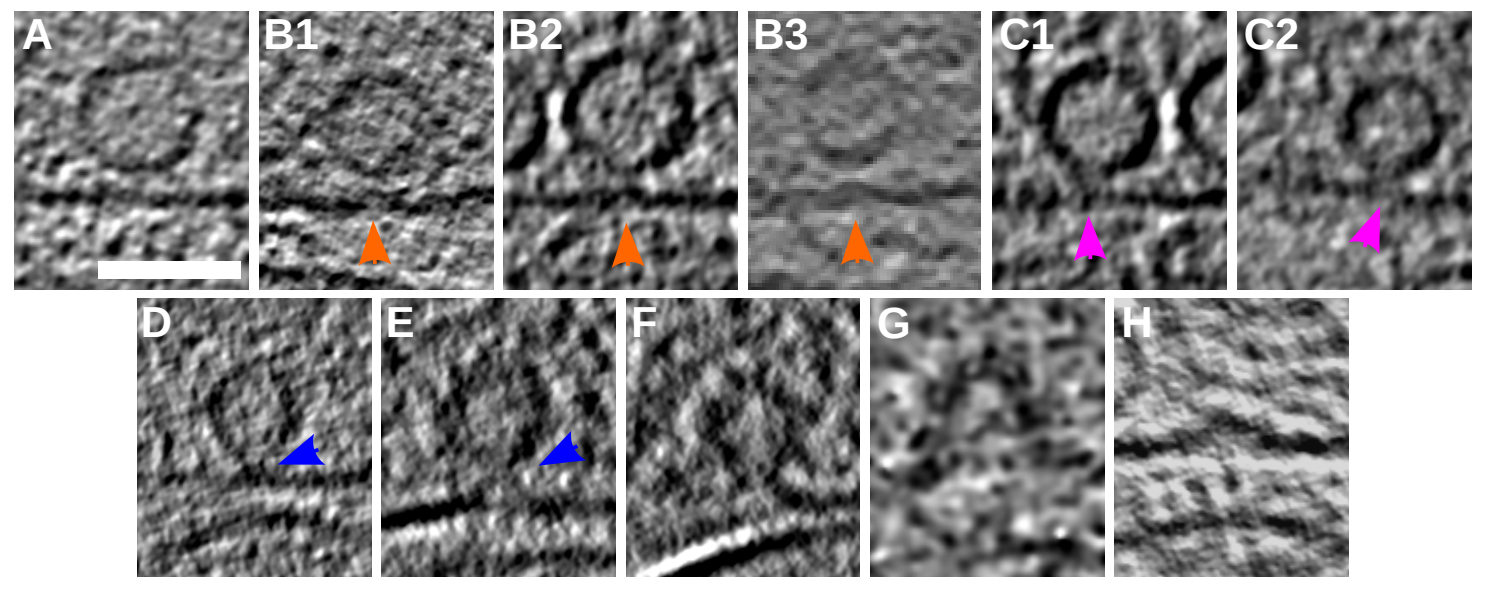

Figure 2: SV exocytosis morphology. Tomographic slice of non-stimulated (A) and stimulated rat synaptosomes (B-H). A) Image of a 2.2-nm thick tomographic slice showing a non-stimulated with SVs at the AZ and a straight PM. B1) Membrane curvature event, 2.2-nm thick tomographic slice. B2) Membrane curvature event, 6.5-nm thick tomographic slice. B3) Membrane curvature event, $2.24 \mathrm{~nm}$ thick tomographic slice. Orange arrows showing membrane curvature event. C1,C2) Lipid perturbations of PM and SV, 22-nm thick tomographic slices. The space between SV and PM is denser than in the non-stimulated synaptosomes (see pink arrow). D-F) Vesicles with a pore opening that might be on the way to full collapse fusion, 33-nm thick tomographic slice thickness: $22 \mathrm{~nm}$ (D), 30.8 (E), $33 \mathrm{~nm}$ (F). G) Wide pore opening, most likely on the way to full collapse fusion, 2.2-nm tomographic slice. H) Remaining bump at the end of full collapse fusion, 11-nm thick tomographic slice. Scale bar, $50 \mathrm{~nm}$.

Stimulated synaptosome datasets were divided into early and late fusion stages, respectively, based on the morphology of SV and AZ PM. Synapses showing membrane bending and direct lipid contact between SV and PM without an open pore were classified as early fusion (Figure 2 B-E). Those with an open pore or a remaining small bump of a fully collapsed vesicle were classified as late fusion (Figure 2F-H).

\section{Synaptic vesicle distribution is impacted by synaptic activity}

Non-sprayed rat synaptosomes as well as WT-SNAP-25 mouse cultured neuron synapses showed typical SV distribution, as observed in previous cryo-ET studies (Figure 3)[13]. Vesicle occupancy in WT- 
SNAP-25 synapses was 0.09 in the most proximal zone ( $0-25 \mathrm{~nm}$ from the AZ PM), and peaked to 0.18 in 25-50 nm zone. It then dropped to 0.08 in the intermediate zone $(50-75 \mathrm{~nm})$ and rose steadily more distally to reach a plateau of $\sim 0.16$ spanning the range of 150 to $250 \mathrm{~nm}$ distance. Finally, SV occupancy gradually decreased as the distance from the AZ increased (Figure $\underline{3} A$ ).

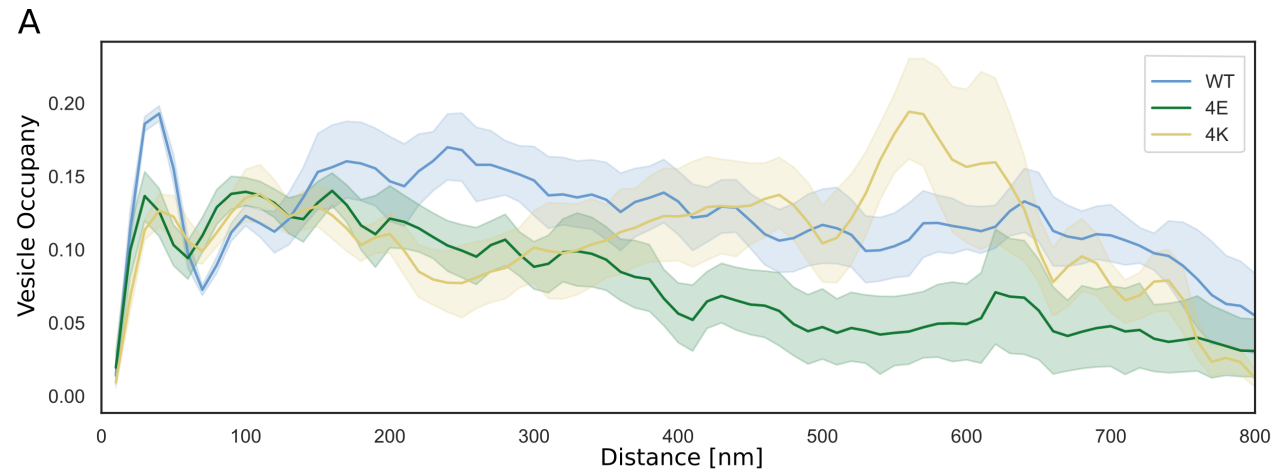

B
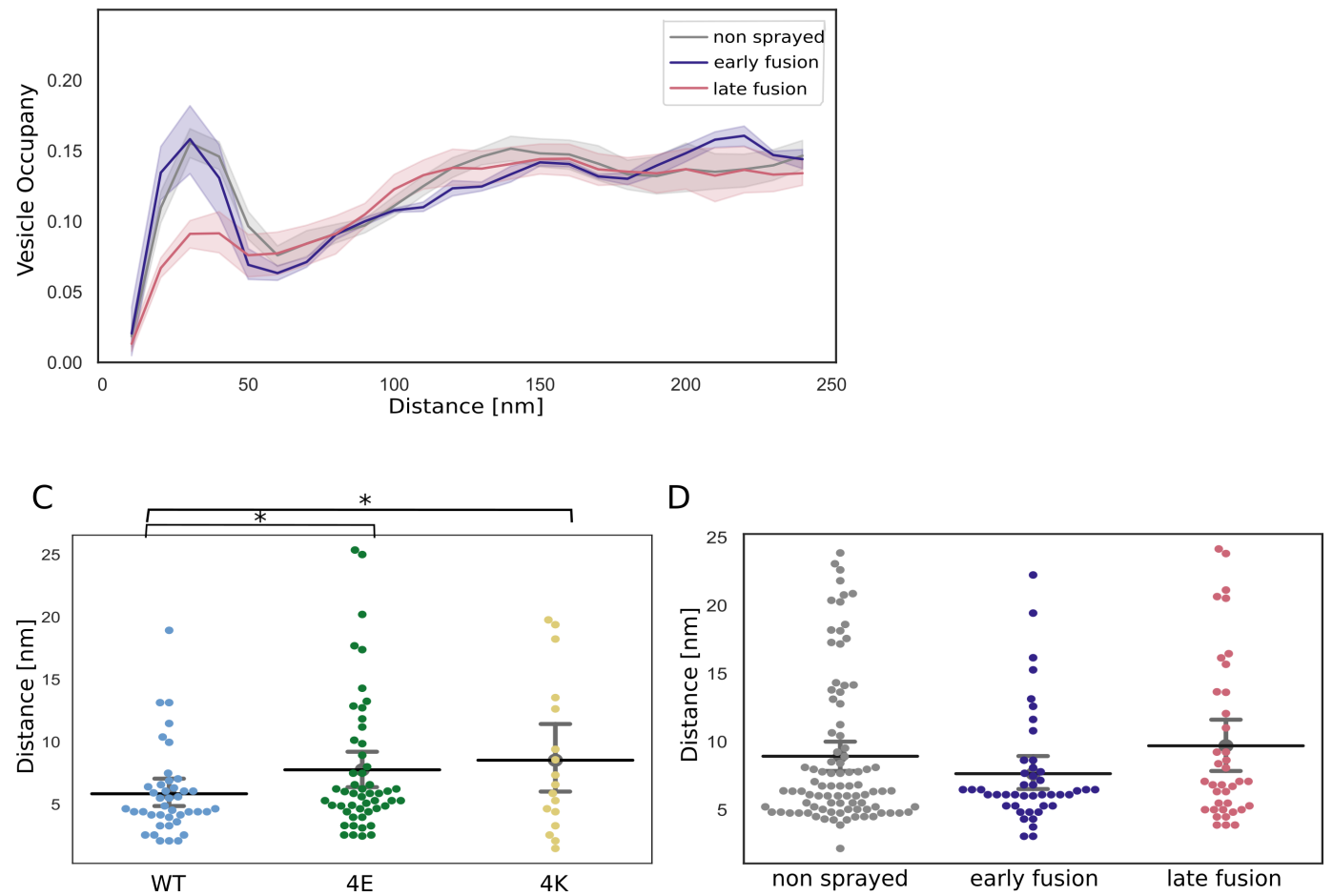

Figure 3: SV distribution. (A, B) Vesicle occupancy expressed as fraction of cytosol volume occupied by vesicles as a function of distance to AZ in (A) cultured neurons and (B) synaptosomes. Each solid line represents the mean occupancy value for the experimental group, while the shaded areas depict the $95 \%$ confidence interval. (C, D) Distance of proximal SVs from the AZ. Horizontal line: mean; whiskers: 95\% confidence interval. Statistical test: t-test.

The absolute values differ between WT cultured mouse neurons and non-stimulated rat synaptosomes but the SV occupancy distribution follows the same pattern. The difference in absolute values can likely be attributed to the different experimental and animal models used. Sprayed synaptosomes that were showing early signs of exocytosis had a nearly identical SV occupancy pattern as non-sprayed synaptosomes (Figure $\underline{3} B$, dark blue and gray, respectively). However, when SV full collapse figures were apparent, SV occupancy in the proximal zone was significantly reduced, whereas SV occupancy further away from the AZ PM was unchanged (Figure $3 B$, red). This is consistent with some membrane proximal SVs having engaged in exocytosis, while none of the recycling and reserve pool SVs have. In order to investigate the consequences of chronic high or low synaptic activity, we investigated the $4 \mathrm{E}$ and $4 \mathrm{~K}$ mutants (Figure $3 \mathrm{~A}$, green and gold, respectively). In the most proximal $50 \mathrm{~nm}$, SV were significantly less concentrated in the constitutively depressed 4E mutant than in the WT. However, they were significantly more abundant between 75 and $100 \mathrm{~nm}$. Furthermore, proximal SVs were on average more distant to the AZ PM in the 4E mutant than in the WT (Figure $\underline{3}$ C). These observations are consistent with the increased distance between SVs and the 
PM induced by the repulsion between negative charges present in SNAP-25 and on the AZ/PM. In the most distal zones, SV occupancy gradually decreased in the 4E mutant and was significantly lower than in the WT over most of the 250 to $750 \mathrm{~nm}$ distance range. The decrease may reflect deleterious effects associated with abnormally low synaptic activity. The 4K mutant displayed a significantly decreased SV occupancy in comparison to the WT in the most proximal $50 \mathrm{~nm}$. 4K proximal SVs were in average more located further away from the AZ PM than WT proximal SVs. Furthermore, proximal SVs were in average more distant to the AZ PM in the $4 \mathrm{~K}$ mutant than in the WT (Figure $3 \mathrm{C}$ ). This can be readily attributed to the high probability of spontaneous exocytosis generated by the additional positive charges of the SNARE bundle. Between $50 \mathrm{~nm}$ and $75 \mathrm{~nm}$ away from the AZ PM, SV occupancy was higher in the $4 \mathrm{~K}$ mutant than in the WT, consistent with recycling pool SVs being recruited. From 100 to $250 \mathrm{~nm}$, SV occupancy dropped steadily, in contrary to the WT, and from a distance of $170 \mathrm{~nm}$, it was significantly lower. Yet, beyond $250 \mathrm{~nm}$, it rose linearly until $450 \mathrm{~nm}$, becoming indistinguishable from WT occupancy, and then experienced a sharp increase, peaking to 0.2 at a distance of $550 \mathrm{~nm}$, significantly higher than WT occupancy, before dropping quickly and significantly lower than WT occupancy.

\section{Proximal vesicles form additional tethers following stimulation}

We investigated the tethering state of proximal SVs (i.e. the SVs whose center is located within $45 \mathrm{~nm}$ of the AZ PM) prior to and following stimulation in synaptosomes. In non-sprayed synaptosomes, 54\% of the proximal vesicles were tethered, which is in agreement with previous results (Supplementary

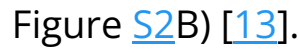

Interestingly, in the early fusion group the fraction of tethered proximal vesicles significantly increased to $80 \%$ ( $P<0.05, \chi 2$ test). In the late fusion group, however, 53\% of the proximal vesicles were tethered, which is not significantly different to the non-sprayed group. The average number of tethers per proximal SV followed the same pattern. Proximal SVs had $0.89 \pm 0.12$ tethers in the nonsprayed group (Figure $4 \mathrm{D}$ ). This parameter rose to $2.09 \pm 0.33$ in the early fusion group, while it returned to $1.00 \pm 0.20$ in the late fusion group.

A

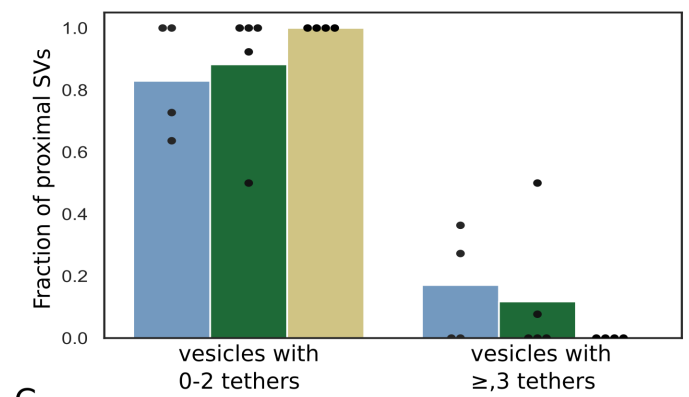

$c$

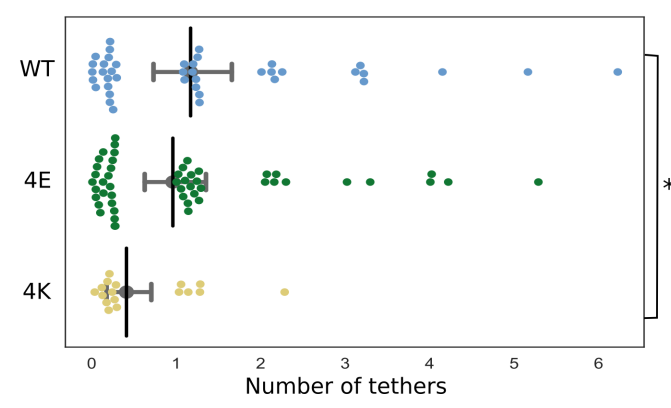

B

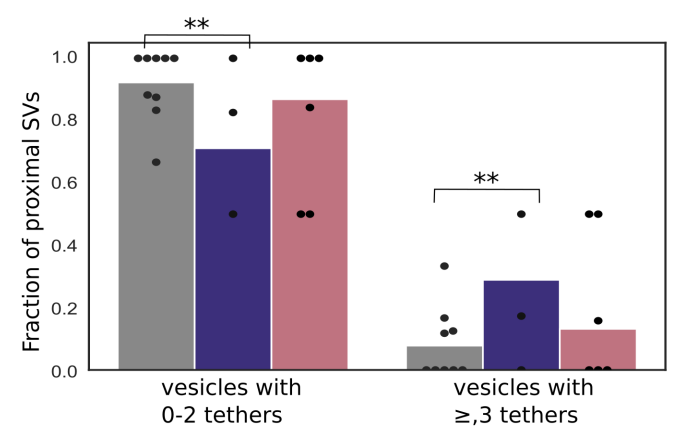

D

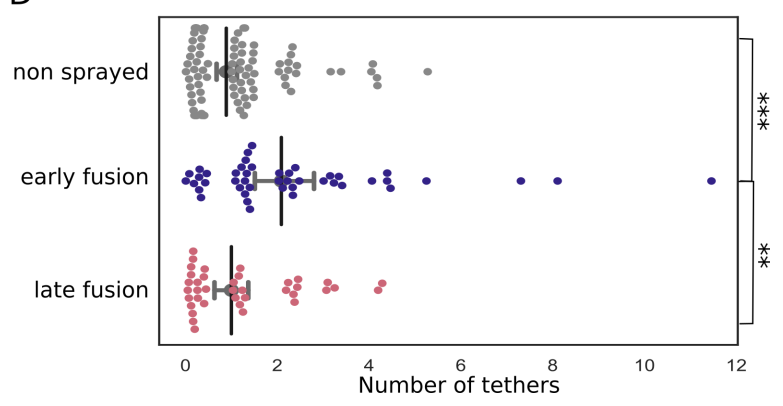

Figure 4: Proximal SV tethering. (A, B) Fraction of proximal SVs that are triple tethered. Each bar shows the overall fraction of all proximal SVs from a given experimental condition. Each dot represents the value of an individual synapse. Statistical test: $\chi^{2}$ test. (C, D) Number of tethers per proximal SV. Each dot represents an individual SV. The vertical line represents the mean value, and the horizontal whiskers correspond to the $95 \%$ confidence interval. Statistical test: $t-$ test. 

made available under aCC-BY 4.0 International license.

We then analyzed whether the decreased occupancy in the late fusion group was associated with a decreased number of triple-tethered SVs, (defined as SV with at least three tethers) which as mentioned in the introduction are suggested to belong to the RRP. In resting, non-sprayed synapses $8 \%$ of the proximal SVs were triple-tethered (Figure $4 \mathrm{~B}$ ). Surprisingly, the fraction of triple-tethered proximal SVs drastically increased to $29 \%$ in the early fusion group $(P<0.001, \chi 2$ test). The fraction decreased to $13 \%$ in the late fusion group. This suggests that upon stimulation some proximal SVs very rapidly acquire new tethers. Using our definition of the RRP (vesicles that are triple-tethered) this would indicate that the RRP rapidly increases after stimulation and more vesicles become primed for exocytosis. Furthermore, the lower proximal vesicle occupancy in the late fusion group indicates that under our stimulation conditions, replenishing vesicles to the proximal zone is slower than their release.

The situation in the WT-SNAP-25 neurons was similar to unstimulated synaptosomes. 53\% of the all proximal SVs were tethered and $17 \%$ of all proximal SVs belonged were triple-tethered (Supplementary Figure $\underline{\mathrm{S}} \mathrm{A}$ and Figure $\underline{4 A}$. On average, proximal SVs had $1.17 \pm 0.23$ tethers. The corresponding values for the $4 \mathrm{E}$ mutants were not significantly different ( $15 \%$ and $0.96 \pm 0.18$, respectively). However, in all $4 \mathrm{~K}$ mutant datasets there was not a single SV that was part of the RRP, i.e. triple-tethered. Consistently, the number of tethers per proximal SV was significantly lower in the $4 \mathrm{~K}$ mutant than in the WT (Figure $4 \mathrm{C}$ ). These results are in line with physiological measurements that have shown that the RRP is depleted in the chronically spontaneously active 4K mutant, and they provide additional evidence that RRP-vesicles have at least 3 tethers. [12].

\section{Synaptic activity modifies inter-SV connectivity}

The majority of SV are linked to other SVs via molecular bridges previously termed connectors connectors $[13,14]$. The function and composition of connectors are not clear yet. It was earlier proposed that connectors limit SV dispersion and allow SV mobilization for release. It is generally assumed that synapsin is involved in connector formation and may be one of its components. It has been suggested that connectors reduce SV mobility and maintain a local high SV concentration in the presynapse. The connectivity level of an individual SV might be one of the factors defining the pool to which the SV belongs. To shed some light on the role of connectors, we analyzed SV connectivity in our datasets. We focused most of our analysis to the SVs located at distance of the AZ PM lower than $250 \mathrm{~nm}$. Furthermore, we defined 4 distance groups: proximal $(0-45 \mathrm{~nm})$, intermediate $(45-75 \mathrm{~nm})$, distal $1(75-150 \mathrm{~nm})$, distal $2(150-250 \mathrm{~nm})$, as in previous studies $[13,31]$. We first analyzed synaptosomes. In non-sprayed synaptosomes datasets, approximately $70 \%$ of the proximal and intermediate SVs were connected to other vesicles. In distal 1 and 2 regions, this value rose to 84 and $87 \%$, respectively. Similarly, the number of connectors per vesicles significantly increased from the proximal region $(1.63 \pm 0.13)$ to the distal 1 region $(2.57 \pm 0.09, \mathrm{P}<0.01)$ and the distal 2 region $(2.78 \pm$ $0.10, P<0.0001$ ) (Figure $\underline{5}$ B). Sprayed early and late fusion synapses showed a similar pattern, with significantly more connectors per SV in distal 1 region than in proximal region for the late fusion group, and more connectors per SV in distal 2 region for both early and late fusion groups. Nonetheless, proximal SV connectivity and number of connectors per SV dropped in the late fusion group to $53 \%\left(P<0.09, \chi^{2}\right.$ test) and $1.12 \pm 0.15(P<0.05)$, respectively (Figure $5 \mathrm{D}$ and $\left.\mathrm{B}\right)$. This decrease originates from tethered SVs. Indeed, $1.65 \pm 0.23$ connectors per tethered SVs were found in the nonsprayed group. This number increased to $2.58 \pm 0.45$ in the early fusion group $(P<0.05)$ and dropped to $0.85 \pm 0.25$ in the late fusion group $(P<0.05)$, whereas no significant differences were found in the numbers of connnectors per proximal non-tethered SVs (Figure $\underline{5} \mathrm{H}$ ). Furthermore, the decrease in proximal connectivity was only observed in non triple-tethered SVs, of which only $51.5 \%$ of the vesicles were connected as opposed to $72.3 \%$ before stimulation $\left(P<0.01, \chi^{2}\right.$ test; Supplementary Figure S2 J). Consistently, the number of connectors per non triple-tethered proximal SV went from $1.64 \pm 0.17$ in the non-sprayed group, rose significantly to $2.69 \pm 0.54$ in the early fusion group $(P<0.05)$ and dropped to $0.9 \pm 0.19$ in the late fusion group $(P<0.05)$ (Figure $\underline{5}$ F). Taken together, our observations indicate that following depolarization, the number of connectors per proximal single- or double-tethered vesicles 
(i.e. non-RRP) first increases and then decreases to a value lower than the initial one. We have seen earlier that the fraction of tethered proximal SVs does not differ between non-sprayed and late fusion synaptosomes. Thus, our data suggest that establishing connectivity is a slower process than tethering. We hypothesize that given the free space made in the proximal region after some SVs have fused, non-connected vesicles from the intermediate region diffuse to the proximal zone and become tethered to the AZ PM. Only subsequently, these newly tethered vesicles get interconnected.

Furthermore, we have observed that connectors remained present between fusing SV and neighbor SV (Supplementary Figure S3). This, in addition to passive diffusion, can contribute to replenishing the RRP.
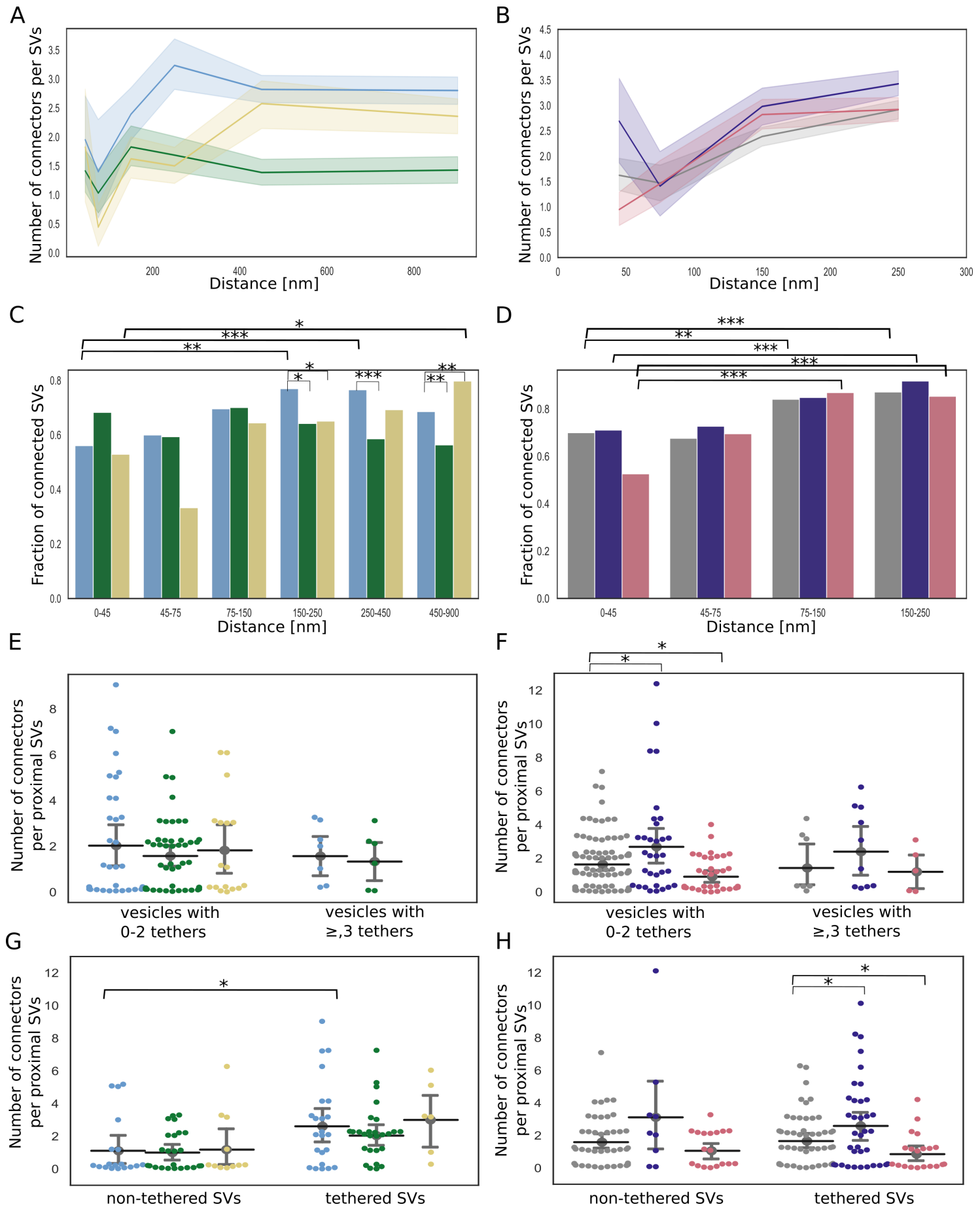

Figure 5: SV connectivity. (A, B) Number of connectors per SV as a function of their distance to the AZ PM for mouse neurons (A) and rat synaptosomes (B) Each solid line represents the average value of all SVs belonging to a particular experimental condition. Shaded areas represent 95\%-confidence interval ranges. (C, D) Fraction of connected vesicles as a function of distance to the AZ PM for mouse neurons (C) and rat synaptosomes (D). (E, F) Number of connectors per proximal SV belonging or not to the RRP for mouse neurons (E) and rat synaptosomes (F). (G, H) Number of connectors per tethered or non-tethered proximal SV for mouse neurons $(G)$ and rat synaptosomes $(H)$. *: $P<0.05$. 
bioRxiv preprint doi: https://doi.org/10.1101/2022.03.07.483217; this version posted March 7,2022 . The copyright holder for this preprint (which was not certified by peer review) is the author/funder, who has granted bioRxiv a license to display the preprint in perpetuity. It is made available under aCC-BY 4.0 International license.

We then analyzed SNAP-25 neurons. For SNAP-25-WT, similarly to non-sprayed synaptosome, the fraction of connected SVs was significantly higher in the distal 2 region than in the proximal region $(p<0.01)$, albeit the absolute values were overall lower than in synaptosomes (Figure $5 \mathrm{C}$ ). Consistently, the number of connectors per SV in SNAP-25-WT synapses increased from $1.95 \pm 0.38$ in the proximal region to $3.23 \pm 0.21$ in the distal 2 region (Figure $\underline{5} \mathrm{~A}, \mathrm{P}<0.01$ ). The fraction of connected $\mathrm{SV}$ s in the distal 2 region was significantly lower in the 4E and $4 K$ mutant than in the WT $(p<0.05)$. This was supported by a significantly lower number of connectors per SV in the distal 1 region for the $4 \mathrm{~K}$ mutant versus the WT $(P<0.05)$, and in the distal 2 region for the 4E mutant versus the WT $(P<0.0001)$ as well as for the $4 \mathrm{~K}$ mutant versus the WT $(P<0.0001$, Figure $\underline{5} \mathrm{~A})$. The number of connectors per proximal SV was not affected by the mutations (Figure $\underline{5} \mathrm{E}$ and $\mathrm{G}$ ). These results indicate that prolonged abnormal exocytotic activity is correlated with severe changes in intervesicular connectivity in the distal region.

\section{Discussion}

Due to its transient nature, SV exocytosis has been difficult to characterize morphologically. A number of questions remain partially unresolved to this date. In particular, it has been suggested that following $\mathrm{Ca}^{2+}$ entry, the insertion of synaptotagmin-1 into the membrane induces an increase in membrane curvature, which lowers the energy barrier of fusion. Such membrane deformations have been observed in biochemically reconstituted models of exocytosis but have not yet been reported in functional synapses [11,32]. Moreover, it is not clear whether the membrane deformation occurs subsequently to $\mathrm{Ca}^{2+}$ influx or if primed SVs and their PM counterpart present such deformation [32]. The optimal sample preservation delivered by cryo-ET makes it possible to investigate the role of tethers located between SVs and the AZ PM and the function of inter-SV connectors. Combining cryoET with spray-mixing plunge-freezing enabled us to investigate the morphological changes occurring immediately after depolarization.

\section{Membrane curvature increases following depolarization}

Depolarization through spraying droplets of $\mathrm{KCl}$ solution on synaptosomes milliseconds before freezing allowed us to capture snapshots of exocytosis (Figure 2B1-B3). We observed that the curvature of some PM regions facing some SVs increased following depolarization. The SV facing such a PM buckling also seemed to get kinked. These deformations were not seen in non-sprayed synaptosomes. This indicates that in functional synapses exocytosis starts with a $\mathrm{Ca}^{2+}$-dependent membrane deformation. The latter may be caused by the intercalation of synaptotagmin-1 C2A and C2B domains between membrane head groups. A recent biophysical study indicated that C2A and C2B preferably insert in SV membrane and PM, respectively [33]. It may also be due to the tension/force induced by SNARE-complex zippering [34]. Subsequent snapshots showed a fuzzy contact point between the SV and the PM, which likely corresponds to lipid splaying or the merging of the two membranes. Membrane fusion then occurred and yielded classical $\Omega$-figures with variable neck diameters. Finally, nearly fully collapsed SVs were imaged. Overall our observations support the standard model of full collapse SNARE-dependent membrane fusion $[35,36]$ and reveal details of exocytosis early stage, prior to actual membrane fusion.

\section{SV local concentration correlates with SV connectivity}

SV local concentration - a.k.a SV occupancy - is tightly correlated with the distance from the AZ PM. Under resting conditions, SV occupancy reaches a local maximum at the distance approximately 25 $\mathrm{nm}$ and a local minimum at approximately $75 \mathrm{~nm}$ from the AZ PM, before rising again with the distance increasing (Figure $3 \mathrm{~A}$ and $\mathrm{B}$ ), in agreement with previous reports [13]. By definition, all SVs in the proximal region are directly facing the PM. Their high concentration can be attributed to the fact that more than $50 \%$ of them are tethered to the PM. On the other hand, the number of connectors 
bioRxiv preprint doi: https://doi.org/10.1101/2022.03.07.483217; this version posted March 7,2022 . The copyright holder for this preprint (which was not certified by peer review) is the author/funder, who has granted bioRxiv a license to display the preprint in perpetuity. It is made available under aCC-BY 4.0 International license.

per SV and SV connectivity gradually increases with the distance from the AZ (Figure $\underline{5} A-D)$. This increase correlates with the increase in occupancy. Thus, we may hypothesize that SV local concentration is a function of their level of tethering to the PM and of connection with other SVs. Interestingly, under short stimulation of a few ms, SV occupancy only decreases in the proximal region, as a consequence of the fusion of SVs with the PM (Figure $3 \mathrm{~B}$ ). In order to further assess the relation between SV tethering, connectivity, and occupancy, we analyzed synapses bearing expressing either WT SNAP-25, a more positively charged mutant (4K), or a more negatively charged mutant (4E) [12]. The $4 \mathrm{~K}$ mutant has a decreased energy barrier to membrane fusion and causes constitutively active exocytosis, whereas the 4E mutant shows a decreased exocytotic activity because of a higher energy barrier to membrane fusion. Both mutants had a significantly decreased proximal SV occupancy (Figure $3 \mathrm{~A}$ ). In the case of the $4 \mathrm{~K}$ mutant, this was probably due to the high frequency of spontaneous exocytosis. On the other hand, the 4E mutant, because of its additive negative charges, tends to repel SVs from the PM (Figure 3 C), which can explain their decreased proximal occupancy. Over a narrow distance range, around $75 \mathrm{~nm}$, SV occupancy of both mutants was significantly higher than that of the WT. Further away, SV occupancy was most significantly lower for the 4E mutants than for the WT. The 4K mutant followed the same trend but had a massive increase of SV occupancy between 500 and $600 \mathrm{~nm}$, before falling again. Interestingly, the number of connector per SV follows a similar pattern as SV occupancy in both mutants. In the 4E mutant, it remains significantly lower than in the WT from approximately $200 \mathrm{~nm}$ and beyond. In the $4 \mathrm{~K}$ mutant, this value is significantly lower than in the WT from approximately $100 \mathrm{~nm}$ but it then rises close to WT values from approximately $400 \mathrm{~nm}$ onward. This increased occupancy may be due to the recycling of spontaneously fusing SVs. Our data show that strong disturbances in exocytotic activity lead to profound differences in SV occupancy and SV connectivity. We note that a correlation exists between SV connectivity and concentration. Future studies will be necessary to assess whether SV concentration depends on the SV connectivity and to decipher the molecular mechanism influencing these parameters.

\section{SNAP-25 4K mutant further supports the RRP morphological definition}

Previously, we showed that the number of tethers of a SV defines whether its exocytosis can be induced by treatment with a hyperosmotic sucrose solution, which corresponds to a definition of the RRP $[13,14]$. We reported that SVs with at least 3 tethers belong to the RRP, according to this definition. In order to further assess this model, we analyzed synapses of neurons expressing the SNAP-25 mutants. $17 \%$ of the WT proximal SVs had 3 tethers or more. Critically, the $4 \mathrm{~K}$ mutant had none such SV. As the RRP (assessed with hyperosmotic sucrose treatment) in this mutant was formerly shown through functional assays to be depleted, our present observation further supports our morphological definition of the RRP [12]. 15\% of the proximal SVs had 3 tethers or more in the 4E mutant, which is very similar to the WT situation, while this mutant was shown to possess a normalsized RRP. Our observations are also consistent with a number of studies that have concluded that SV exocytosis requires a minimum of three SNARE complexes $[8, \underline{9}, 10]$.

\section{Depolarization rapidly induces additional tethering in proximal vesicles}

We compared SV tethering before and shortly after depolarization. Our observations are schematically summarized in Figure $\underline{6}$. Interestingly, the fraction of proximal SVs that were tethered increased by $50 \%$ shortly after stimulation, in synapses showing early signs of exocytosis. Simultaneously, the number of tethers per proximal SV more than doubled and, the fraction of proximal SVs with 3 or more tethers tripled. In presynaptic terminals presenting more advanced stages of exocytosis ( $\Omega$-figures), all these measurements returned to pre-stimulation values. These data indicate that immediately after the onset of stimulation a quick and massive increase in tethering occurs. This phenomenon was resolved in our measurements, because the spraying of synaptosomes with an intermediate $\mathrm{K}^{+}$-concentration made it possible to isolate synaptosomes in an early stage of 
bioRxiv preprint doi: https://doi.org/10.1101/2022.03.07.483217; this version posted March $7,2022$. The copyright holder for this preprint (which was not certified by peer review) is the author/funder, who has granted bioRxiv a license to display the preprint in perpetuity. It is made available under aCC-BY 4.0 International license.

fusion, which would have been missed during either strong or chronic stimulation, which would deplete primed vesicles.

The phenomenon of rapid, depolarization-induced tethering leads to some free proximal SVs becoming tethered to the AZ PM, while some previously single- or double-tethered SVs gained the additional tether(s) that according to our definition of the RRP (as triple-tethered vesicles) would be expected to render them releasable [13]. There are several important implications of this finding. First, the increase of the number of tethers during the initial membrane contact - in excess of the three tethers formed during priming - might help overcome the fusion barrier. Functional

reconstruction led to the suggestion that SNARE-complexes primarily form downstream of $\mathrm{Ca}^{2+}{ }_{\text {-influx }}$ [37], whereas mutagenesis studies in cells supported the notion that SNARE-complexes had already formed before arrival of the $\mathrm{Ca}^{2+}$-trigger, i.e. during priming [38]. In fact, both notions might be partly correct, as the formation of a low number of SNARE-complexes might lead to a stable primed state, defined by a valley in the energy landscape due to the dual inhibitory/stimulatory features of the SNARE-complex [12,39], whereas more SNARE-complexes might form dynamically after triggering, during membrane fusion itself. Accordingly, in in vitro fusion assays additional SNARE-complexes, above those required for fusion pore formation, leads to fusion pore stabilization and release of larger cargos $[10,40]$. Second, vesicles that have not formed three tethers before stimulation might fuse with delayed kinetics during triggering, which accounts for the variable exocytosis kinetics among SVs $[41,42,43,44]$. Superprimed vesicles are expected to have formed the largest number of tethers before stimulation $[41,42]$. Third, overlapping protein complexes might be involved in priming and triggering, depending on the timing of their formation. Accordingly, triggering that stimulates tetherformation might also stimulate priming for those vesicles that were not tethered before stimulation. Indeed, a number of recent publications have suggested that some SVs can get primed extremely quickly in response to $\mathrm{Ca}^{2+}$ influx $[44, \underline{45}, \underline{46}, \underline{47}, \underline{48}]$.
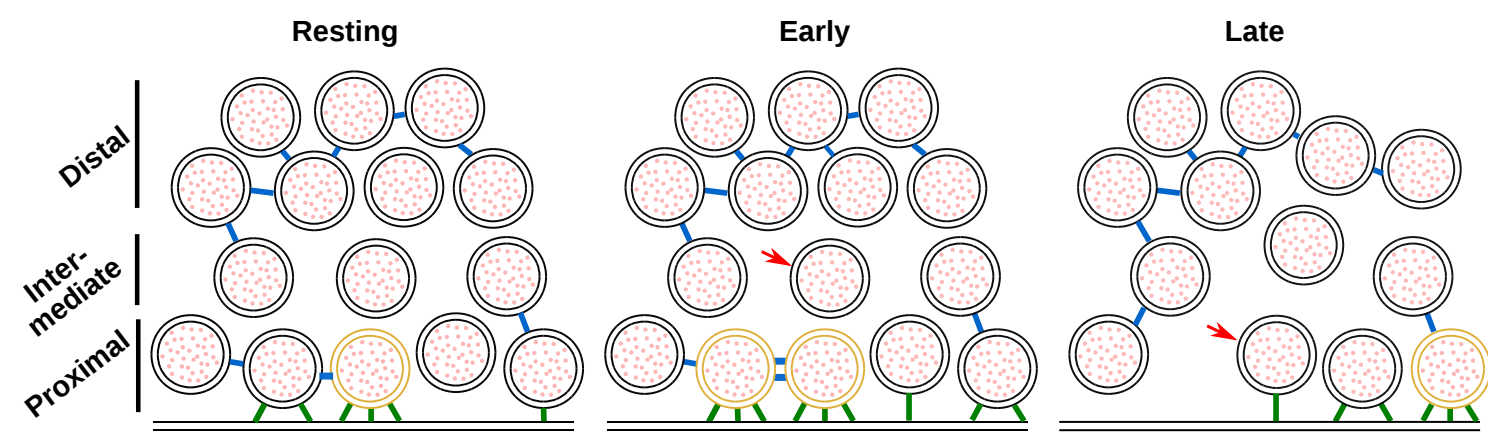

Figure 6: Model depicting a synapse transitioning from resting state to early and late fusion states. Tethering and connectivity changes upon synapse stimulation are depicted. Proximal non triple-tethered vesicles (black proximal SVs) gain additional tethers and some of them become triple-tethered (yellow SVs) shortly after stimulation. Primed vesicles then fuse with the plasma membrane (late fusion) and leave an empty space in the AZ cytoplasm. The number of connectors (depicted in blue) per proximal SV increases in late fusion tripled-tethered vesicles. The red arrow shows a vesicle initially located in the intermediate region, which diffuses to the proximal region in the late fusion state. Tethers are shown in green.

\section{Conclusion}

Our study revealed fine morphological changes occurring in the presynaptic terminal immediately after the onset of exocytosis, as well as in chronically active or inactive synapses. It indicates increased SV tethering induced the rise in presynaptic $\mathrm{Ca}^{2+}$, potentially corresponding to SV superpriming, and preceding SV fusion. It also highlights modifications of proximal SV interconnections in response to evoked exocytosis, as well as more drastic modifications of distal SV interconnections in chronically active synapses and in inactive synapses. These changes likely affect SV mobility and recruitement at the AZ.

\section{Materials and methods}




\section{Constructs and viruses}

SNAP-25B was N-terminally fused to GFP and cloned into a pLenti construct with a CMV promoter [49]. Mutations were made using the QuikChange II XL kit (Agilent). The mutations were verified by sequencing and have been published before [12]. The preparation of lentiviral particles followed standard protocols.

\section{Animals}

Synaptosomes were prepared from adult male or female Wistar rats obtained from the central animal facilities of the Department of Biomedical Research of the University of Bern. Adult male or female Wistar rats at an age of 6-8 weeks were slightly stunned by $\mathrm{CO}_{2}$ and quickly decapitated with a guillotine. The procedures used were in accordance with the Swiss Veterinary Law guidelines. Heterozygous SNAP-25 KO C57/BI6-mice were routinely backcrossed to BI6 to generate new heterozygotes. The strain was kept in the heterozygous condition and timed heterozygous crosses and caesarean section were used to recover knockout embryos at embryonic day 18 (E18). Pregnant females were killed by cervical dislocation; embryos of either sex were collected and killed by decapitation. Permission to keep and breed SNAP-25 mice was obtained from the Danish Animal Experiments Inspectorate and followed institutional guidelines as overseen by the Institutional Animal Care and Use Committee (IACUC). Newborn (P0-P2) CD1 outbred mice of either sex were used to create astrocytic cultures and for that were killed by decapitation.

\section{Synaptosome preparation}

Rat synaptosomes were prepared as previously described [50], with some modifications. The cerebral cortex and the hippocampi were removed in sucrose buffer (SEH: $0.32 \mathrm{M}$ sucrose, $1 \mathrm{mM}$ EDTA, $10 \mathrm{mM}$ HEPES; HEPES, \#H4034, Sigma-Aldrich Corporate Offices. St. Louis, MO, USA) on ice. Homogenization of the tissue was done in SEH with a Potter-Elvehjem grinder (\#358011, Wheaton. Millville, New Jersey, USA), four strokes at the bottom and 6 from top to bottom were applied to the tissue at a speed of 800 turns/min as described in [50]. The whole process from decapitation to homogenization was done within 2-3 min, to obtain functional synaptosomes. Homogenized tissue was then centrifuged at 1000 $\mathrm{g}$ for $10 \mathrm{~min}$ at $4^{\circ} \mathrm{C}$ to remove meninges and blood vessels. The resulting supernatant containing synaptosomes, but also gliosomes and mitochondria was then added to a discontinuous, isoosmotic Percoll (\#P1644, Sigma) gradient with 5\%, 10\% and 23\% in $0.32 \mathrm{M}$ sucrose, $1 \mathrm{mM}$ EDTA in centrifuge tubes (\#344060, Beckman Coulter). The samples were spun in an ultracentrifuge (rotor: SW 40 Ti; Beckman Coulter. Nyon, Switzerland) at $16400 \mathrm{rpm}$ for $12 \mathrm{~min}$ at $4^{\circ} \mathrm{C}$. The layer with the highest amount of functional synaptosomes was between 10-23 \% [50]. The layer was carefully taken out and diluted 1:10 in HEPES buffered medium (HBM; $140 \mathrm{mM} \mathrm{NaCl}, 5 \mathrm{mM} \mathrm{KCl}, 5 \mathrm{mM} \mathrm{NaHCO} 3,1.2 \mathrm{mM}$ $\mathrm{Na}_{2} \mathrm{HPO}_{4}, 1 \mathrm{mM} \mathrm{MgCl}, 10 \mathrm{mM}$ Glucose, $\left.20 \mathrm{mM} \mathrm{HEPES}\right)$. The obtained solution was further spun with an ultracentrifuge (rotor $45 \mathrm{Ti}$; Beckman Coulter) at $11200 \mathrm{rpm}$ for $20 \mathrm{~min}$ at $4^{\circ} \mathrm{C}$. The pellet was carefully and quickly aspirated with a Pasteur pipette to avoid mixture with the solution and then diluted in HBM.

\section{Preparation of astrocytic and neuronal culture}

The procedure has been published before [51]. Glial cells were ready to be used after 10 days. Once they were triturated and counted with a Buerker chamber, 100,000 cells/ml were plated onto untreated 12-well plates containing DMEM supplemented with 10\% foetal bovine serum (FBS), 10000 IU penicillin, $10 \mathrm{mg}$ streptomycin and 1\% MEM non-essential amino acids (DMEM+10\% FBS).

Astrocytes were isolated from CD1 outbred mice (P0-P2). Pups were killed by decapitation and heads were placed in HBSS-HEPES medium (HBSS supplemented with $1 \mathrm{M}$ HEPES). The cortices were isolated 
bioRxiv preprint doi: https://doi.org/10.1101/2022.03.07.483217; this version posted March 7,2022 . The copyright holder for this preprint (which was not certified by peer review) is the author/funder, who has granted bioRxiv a license to display the preprint in perpetuity. It is made available under aCC-BY 4.0 International license.

from the brains and the meninges were removed (dura, pia and arachnoid mater). The cortices were chopped into smaller fragments and transferred to a tube containing $0.25 \%$ trypsin dissolved in Dulbecco's MEM (DMEM). Fragments were incubated for $15 \mathrm{~min}$ at $37^{\circ} \mathrm{C}$. Subsequently, inactivation medium (12.5 mg albumin + $12.5 \mathrm{mg}$ trypsin-inhibitor in DMEM+10\% FBS) was added and the tissue washed with HBSS-HEPES. Tissue was triturated until a smooth cloudy suspension appeared. Cells were plated in $75 \mathrm{~cm}^{2}$ flasks with pre-warmed DMEM, one hemisphere per flask, and stored at $37^{\circ} \mathrm{C}$ with $5 \% \mathrm{CO}_{2}$. Glial cells were ready to be used after 10 days. Glial cells were washed with pre-warmed HBSS-HEPES. Trypsin was added and the flasks were incubated at $37^{\circ} \mathrm{C}$ for $10 \mathrm{~min}$. Cells were triturated and counted with a Buerker chamber before plating 100,000 cells/ml on untreated 12-well plates containing DMEM+10\% FBS. After 2 days, neurons were plated.

Hippocampal neurons were isolated from E18 SNAP-25 KO of either sex. The SNAP-25 KO pups were obtained by pairing two heterozygote animals, and the embryos were recovered at E18 by caesarean section. Pups were selected based on the absence of motion after tactile stimulation and bloated neck [52]; the genotype was confirmed by PCR in all cases. The pups were killed by decapitation and heads were put in HBSS-HEPES medium. The cortices were isolated from the brains and the meninges were removed. The hippocampi were cut from the cortices before being transferred to a tube containing $0.25 \%$ trypsin dissolved in HBSS-HEPES solution. Fragments were incubated for 20 min at $37^{\circ} \mathrm{C}$. Afterwards, the tissue was washed with HBSS-HEPES. The hippocampi were triturated and the cell count was determined with a Buerker chamber. $20 \mu \mathrm{l}$ of solution containing 250,000 cells $/ \mathrm{ml}$ were plated onto the flame sterilized gold R2/2 or R2/1 EM grids as previously described in [51]. Following a 30-min incubation at $37^{\circ} \mathrm{C}$, the grid was transferred into the 12-well plate containing the astrocytes and medium was replaced with NB medium (Neurobasal with 2\% B-27, 1 M HEPES, 0.26\% lutamax, $14.3 \mathrm{mM} \beta$-mercaptoethanol, $10000 \mathrm{IU}$ penicillin, $10 \mathrm{mg}$ streptomycin) for the E18 pups. Between $4 \mathrm{~h}$ and 1 day later, lentiviral particles carrying either SNAP-25-WT, SNAP-25-4E, or SNAP-25-4K constructs were added to the culture [12]. The cultures were incubated for 12 to 14 days before being plunge frozen.

\section{Plunge freezing and spray-mixing}

Rat synaptosomes were prepared for plunge freezing and spray-mixing as follows. The following steps from incubation to plunge freezing were all done at room temperature (RT), equivalent to $23-25^{\circ} \mathrm{C}$. The synaptosomal solution was incubated with calcein blue AM (\#C1429, Molecular Probes-Thermo Fisher Scientific. Waltham, MA, USA) 30 min prior to plunge freezing to visualize the cytosol of functional - esterase containing - cellular compartments such as synaptosomes. Additionally, $1.3 \mathrm{mM}$ $\mathrm{CaCl}_{2}$ and $10 \mathrm{~nm}$ gold fiducials were added (gold fiducials, \#s10110/8. AURION Immuno Gold Reagents \& Accessories. Wageningen, The Netherlands). $\mathrm{CaCl}_{2}$ is necessary to trigger exocytosis and gold fiducials are important to align the acquired tilt series for tomogram reconstruction. The sprayed solution contained $1 \mathrm{mM} \mathrm{CaCl}_{2}$ and $52 \mathrm{mM} \mathrm{KCl}$ in $\mathrm{HBM}$ to depolarize synaptosomes and trigger exocytosis. It also contained fluorescein (\#46955, Sigma) to trace the spray droplets on the EM grid in cryo-FM. The synaptosomal solution was applied to a 200-mesh lacey finder carbon film grid (\#AGS166-H2. Agar Scientific. Elektron Technology UK Ltd. Stansted, UK). Excess liquid on the grid was removed by blotting with a filter paper and the grid was immediately plunge frozen in liquid ethane with a homebuilt plunge freezer and was sprayed on the fly. The plunge freezer and the spraying device (atomizer) were computer controlled with a LabView script (National Instruments Corporation. Mopac Expwy Austin, TX, USA). The spraying device was set similarly to the device in [30]. Nitrogen gas pressure necessary to drive spraying was set to 2.5 bar. The grid was set to pass in front of the spray nozzle at a distance of 3-4 mm. The plunge freezer was accelerated to $0.75 \mathrm{~m} / \mathrm{s}$ and the minimum spray delay was $\sim 7 \mathrm{~ms}$. The atomizer sprays scattered droplets of various size on the EM grid. During the time lapse between spraying and freezing the content of the droplets spreads by diffusion. $\mathrm{KCl}$ diffuses approximately $4 x$ faster than fluorescein. Cryo-ET imaging was done within the diffusion distance of $\mathrm{KCl}$ but outside of the visible spray droplet because the center of the spray droplet would usually be too thick for imaging. This reduces the effective stimulation duration to anything between 0 
bioRxiv preprint doi: https://doi.org/10.1101/2022.03.07.483217; this version posted March 7,2022 . The copyright holder for this preprint (which was not certified by peer review) is the author/funder, who has granted bioRxiv a license to display the preprint in perpetuity. It is made available under aCC-BY 4.0 International license.

$\mathrm{ms}$ and less than the given spray-freeze delay. Moreover, through diffusion, $\mathrm{KCl}$ concentration rapidly rises and then decreases. Hence synaptosomes are not permanently depolarized.

After 12 to 14 days of incubation grids with mouse neurons were plunge frozen with a Vitrobot (Thermofisher Scientific, Mark IV) with a blot time of $3 \mathrm{~s}$ and a blot force of -10 . Wait time and drain time were not used. Humidity was set to $100 \%$ at $4^{\circ} \mathrm{C} .4 \mu$ l undiluted $10 \mathrm{~nm}$ BSA gold tracer (Aurion) was added directly onto the grid prior to plunge freezing.

\section{Cryo-fluorescence microscopy}

After plunge freezing, rat synaptsome samples were imaged at the fluorescent microscope under cryo conditions, with a Zeiss Axio Scope.A1, equipped with an AxioCam MRm camera (Carl Zeiss AG, Germany), and a fluorescence lamp (HXP 120 C). The correlative microscopy stage (\#CMS196, Linkam Scientific Instruments, UK) was cooled down to $-190^{\circ} \mathrm{C}$ by liquid nitrogen and the frozen EM grid was placed into the chamber of the cryostage on a bridge that was not submerged in liquid nitrogen and was close to the objective, where the temperature was around $-150^{\circ} \mathrm{C}$. The filter set used for imaging fluorescein was \#38 (\#000000-1031-346, Zeiss) (BP 470/40, FT 495, BP 525/50; corresponds to GFP) and the one for calcein blue AM was \#49 (\#488049-9901-000, Zeiss) (G 365, FT 395, BP 445/50; corresponds to DAPI). The objective used was either a 10x (\#420941-9911, NA =0.25 Ph1, Zeiss) or a 50x (\#422472-9900, NA = 0.55 Dic, Zeiss), the acquisition software used was AxioVision (AxioVs40x64 V 4.8.3.0, Zeiss) and the processing software was ZEN lite (Zeiss).

\section{Cryo-electron microscopy}

Following cryo-FM, the rat synaptosome grids were mounted in a cryo-holder (Gatan, Pleasonton, CA, USA) and transferred to a Tecnai F20 (FEl, Eindhoven, The Netherlands) which was set to low dose conditions, operated at $200 \mathrm{kV}$, and equipped with a field emission gun. Images were recorded with a $2 \mathrm{k} \times 2 \mathrm{k}$ CCD camera (Gatan) mounted after a GIF Tridiem post-column filter (Gatan) operated in zeroloss mode. The sample was kept at about $-180^{\circ} \mathrm{C}$. Tilt series were acquired using SerialEM [53] for automated acquisition recorded typically from $-50^{\circ}$ to $50^{\circ}$ with a $2^{\circ}$ angular increment and an unbinned pixel size of 0.75 or $1.2 \mathrm{~nm}$. Due to sample thickness (400-700 nm), tomograms were usually not recorded with higher tilt angles. Defocus was set between -8 to $-12 \mu \mathrm{m}$ and the total electron dose used was about 80-100 e $/ \AA^{2}$. Some tomograms were acquired at a Titan Krios equipped with a K2 direct electron detector (Gatan) without energy filter. The K2 camera was operated in superresolution counting mode and between 8-40 frames per tilt angle were taken. Tilt series were acquired using the Latitude software (Gatan) for automated acquisition recorded typically from $-60^{\circ}$ to $60^{\circ}$ with a $2^{\circ}$ angular increment and an unbinned pixel size of $0.6 \mathrm{~nm}$. Defocus was set between -8 to $-12 \mu \mathrm{m}$ and the total electron dose used was about $80-100 \mathrm{e}^{-} / \AA^{2}$. Prior to image processing the frames at each tilt angle, frames were aligned and averaged in 2dx MC_Automator [54] with motioncor [55]. 3D reconstruction was done in IMOD [56]. The alignments were done using the automated fiducial tracking function and the 3D reconstructions were done using the weighted back projection followed by a nonlinear anisotropic diffusion (NAD) filtering. Following tomogram reconstruction only synaptosomes that fulfilled the following criteria were used: 1) even and non-broken PM, 2) synaptic cleft still attached to the presynapse, 3) spherical vesicles, and 4) a mitochondrion in the presynapse necessary to cover the energy demands of the synapse. These criteria indicate that the synaptosome is functional [57].

Cultured mouse neurons tilt series were acquired at a Titan Krios, equipped with a Falcon 3 direct electron detector (Thermofisher Scientific) without energy filter. The Falcon camera was operated in linear mode. Tilt series were acquired using the TEM Tomography software (TFS) for automated acquisition recorded typically from $-60^{\circ}$ to $60^{\circ}$ with a $2^{\circ}$ angular increment and an unbinned pixel size 
of $0.37 \mathrm{~nm}$. Defocus was set between -6 to $-10 \mu \mathrm{m}$ and the total electron dose used was about 80-100 $\mathrm{e}^{-} / \AA^{2}$. Tomogram reconstruction was done as for synaptosome datasets.

\section{Manual and automatic segmentation procedures}

Manual segmentation of SVs, mitochondria, and the active zone PM was done in IMOD (Supplementary Figure \{fig:suppl_tomogram_slices\}B and D, Supplementary Movies S1 and S2). The boundary marked the region to be analyzed by Pyto [58]. The analysis by Pyto was essentially the same as described previously [13] [58]. In short, the segmented area is divided in 1 voxel thick layers parallel to the active zone for distance calculations. A hierarchical connectivity segmentation detects densities interconnecting vesicles (so-called connectors) and densities connecting vesicles to the active zone PM (so-called tethers) (Supplementary Figure \{fig:suppl_tomogram_slices\}B and D, Supplementary Movies S1 \& S2). Distance calculations are done with the center of the vesicle. Mainly default settings were used. The segmentation procedure is conservative and tends to miss some tethers and connectors because of noise. Consequently, the numbers of tethers and connectors should not be considered as absolute values but rather to compare experimental groups. All tomograms analyzed by Pyto were obtained on the same microscope with the same tilt range. The margin of error for false negatives and positives was found to be less than $10 \%$ by comparison with ground truth [58]. As it was done before, an upper limit was set between 2100 and $3200 \mathrm{~nm}^{3}$ on segment volume. The tomograms that were used for this analysis were binned by a factor of 2 to 3 , resulting in voxel sizes between 2.1 and $2.4 \mathrm{~nm}$. Tether and connector length were calculated using the midpoint method [58]. From the stimulated synaptosomes only those that showed visible signs of exocytosis were used for analysis in Pyto.

\section{Data analysis}

If not stated otherwise data in the text are described as mean \pm standard error of the mean (SEM). Wherever possible, data were presented as box plots with the following settings: orange bar, median; box extremities, lower and upper quartiles; whiskers extend up to $1.5 \mathrm{x}$ interquartile range; dots, outliers. We used the same statistical tests as in $[13,31]$. For normal distributed data, the Student's ttest was used. In addition, for data that required to be split into discrete bins (e.g. fraction of connected vesicles by distance to active zone), the $\chi 2$ test was used. To calculate the correlation coefficient for paired samples (such as vesicle distance to active zone and number of tethers), Spearman's rank correlation was used ( $\rho$-test). The confidence values were calculated using two-tailed tests and were indicated in the graphs by , $P<0.05 ;, P<0.01 ;, P<0.001$. We did not apply statistical methods to predetermine sample size but similar sample sizes as previously reported have been used [13]. It was not necessary to apply randomization.

\section{Manuscript preparation}

The manuscript was written with the open and collaborative scientific writing package Manubot [59]. The source code and data for this manuscript are available at https://github.com/aseedb/synaptic tomo ms. 
bioRxiv preprint doi: https://doi.org/10.1101/2022.03.07.483217; this version posted March 7, 2022. The copyright holder for this preprint

(which was not certified by peer review) is the author/funder, who has granted bioRxiv a license to display the preprint in perpetuity. It is made available under aCC-BY 4.0 International license.

\section{Supplementary Material}

\section{Supplementary Figures}
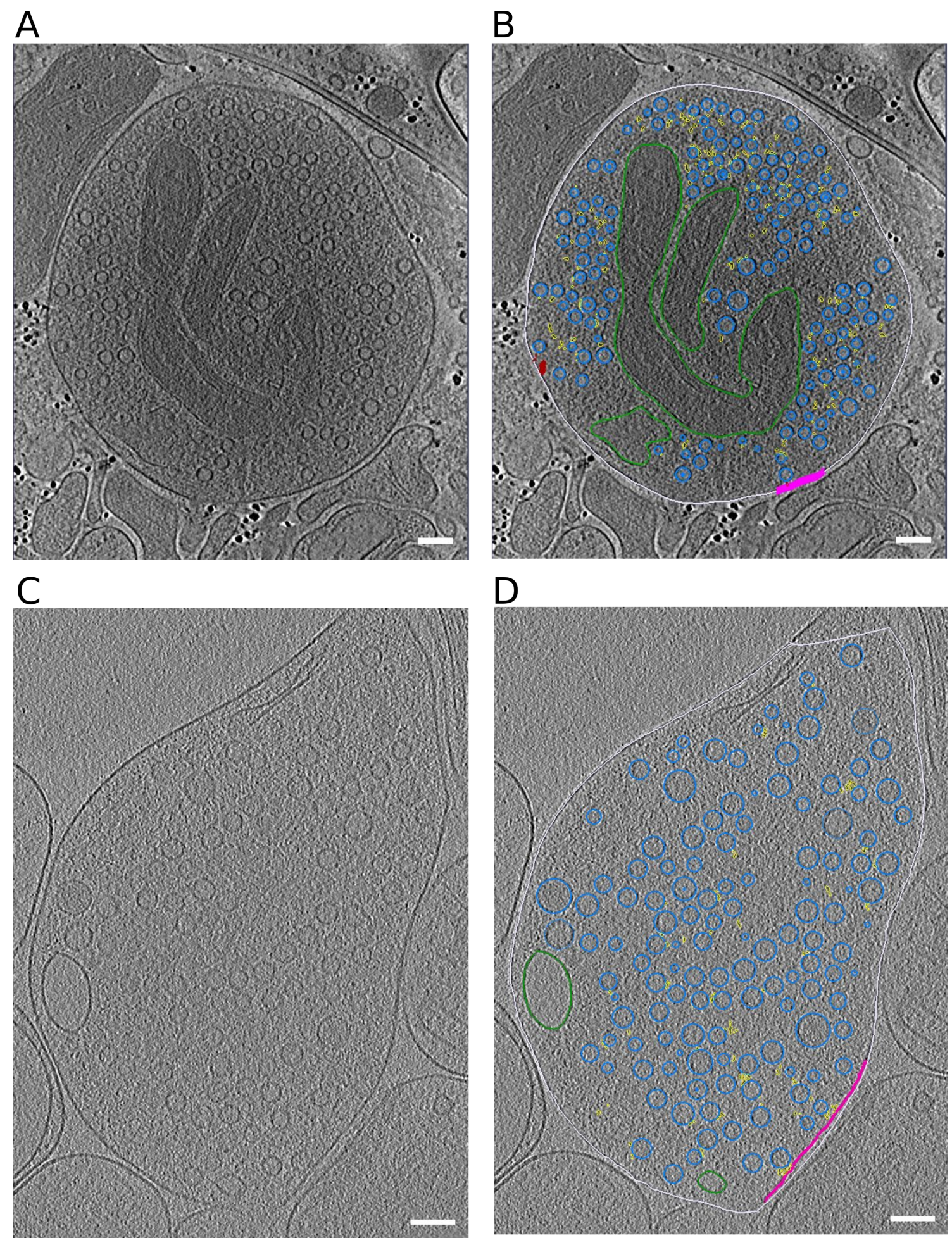

D

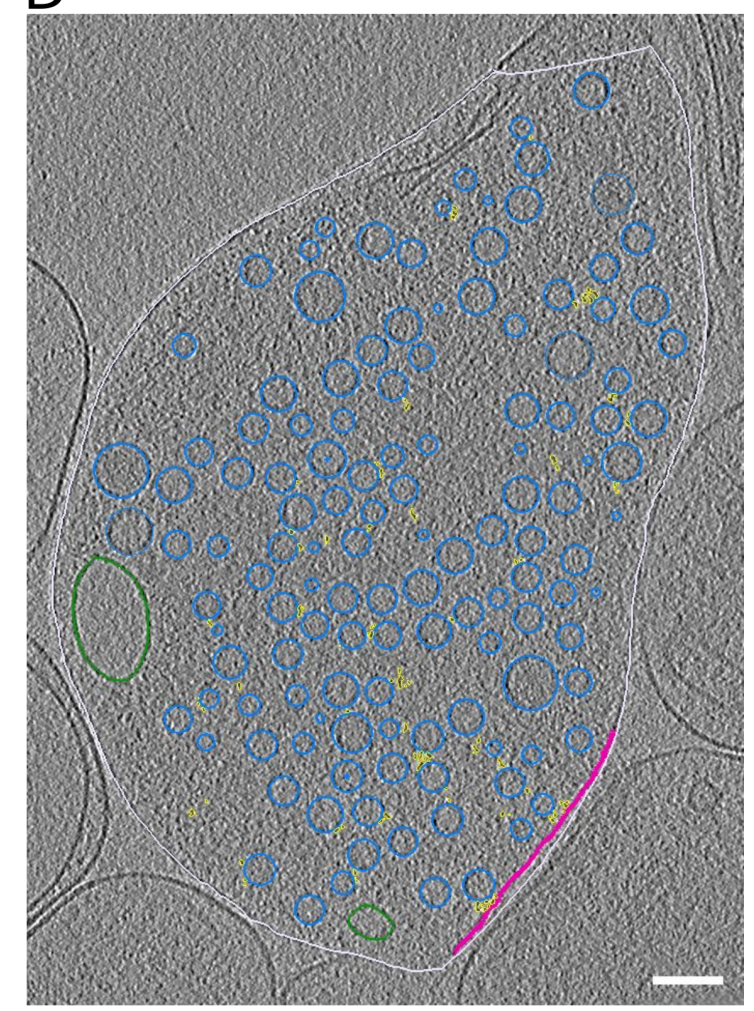

Figure S1: (A, B) Tomographic slice without (A) and with (B) segmentation of synaptosome with late fusion events. (C,D) Tomographic slice without $(C)$ and with (D) segmentation of WT SNAP-25 neurons. Segmentation colors: off-white $=$ cell outline; pink = active zone; blue = synaptic vesicles; green = mitochondria; yellow = connectors, red = tethers Scale bar, $100 \mathrm{~nm}$ 
bioRxiv preprint doi: https://doi.org/10.1101/2022.03.07.483217; this version posted March 7, 2022. The copyright holder for this preprint (which was not certified by peer review) is the author/funder, who has granted bioRxiv a license to display the preprint in perpetuity. It is made available under aCC-BY 4.0 International license.

A

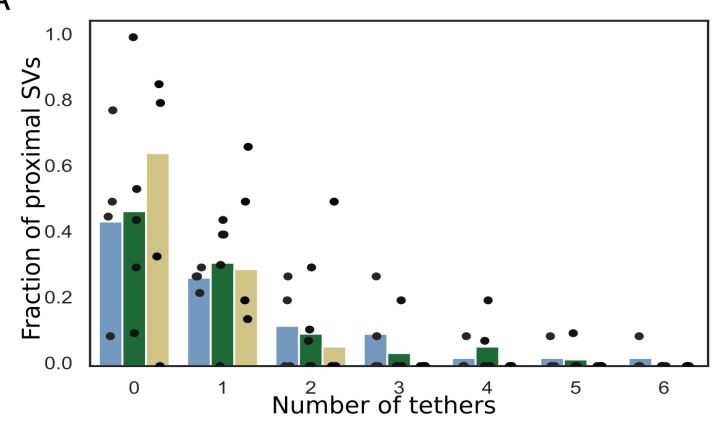

$c$

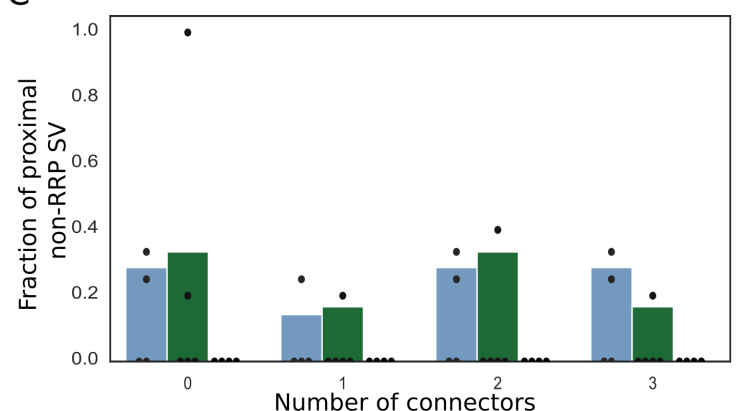

E

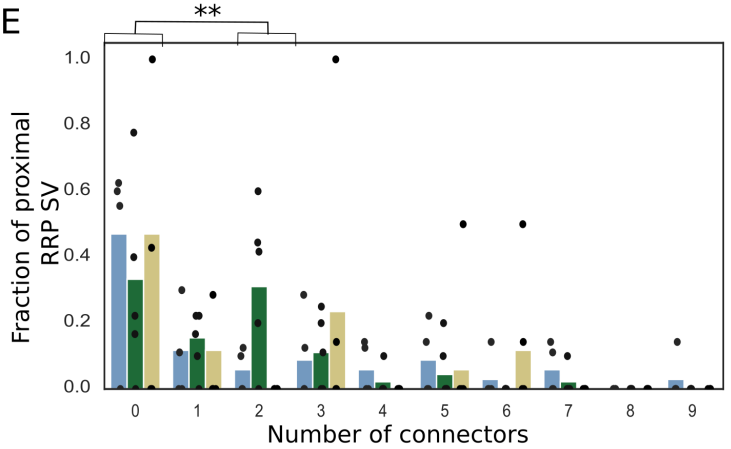

G

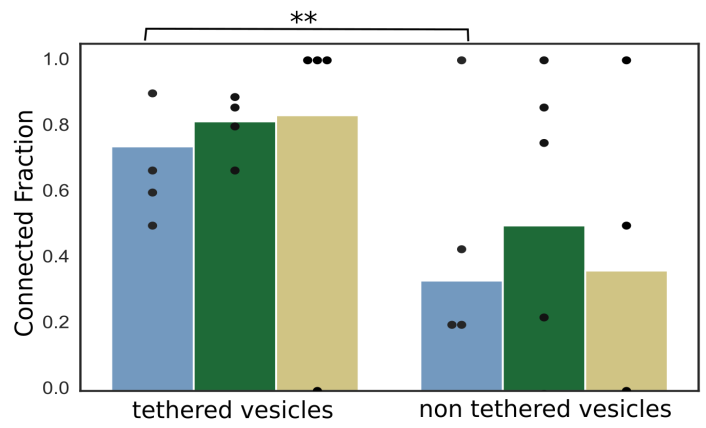

I

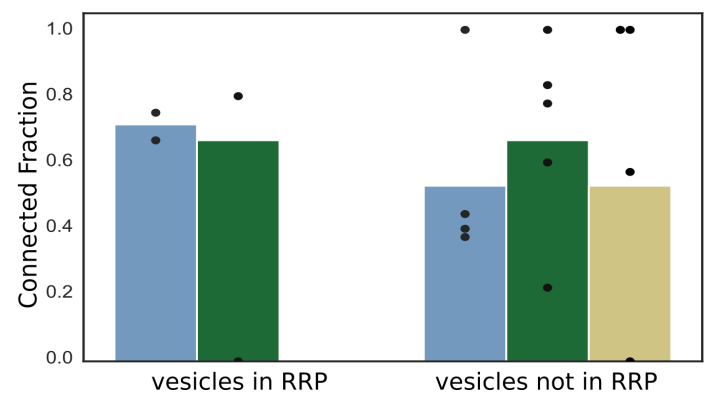

B

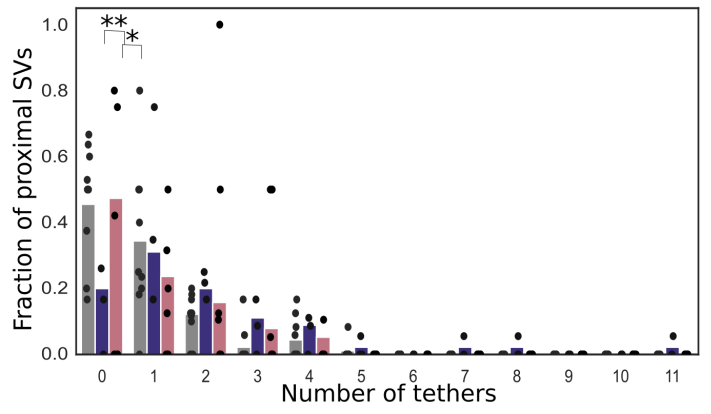

D

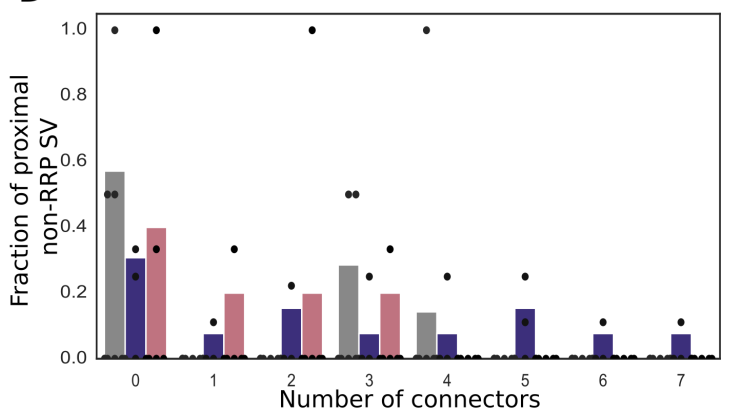

$\mathrm{F}$

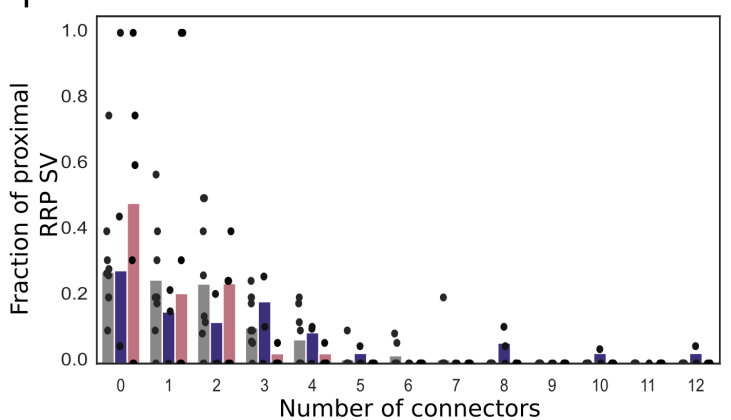

$\mathrm{H}$

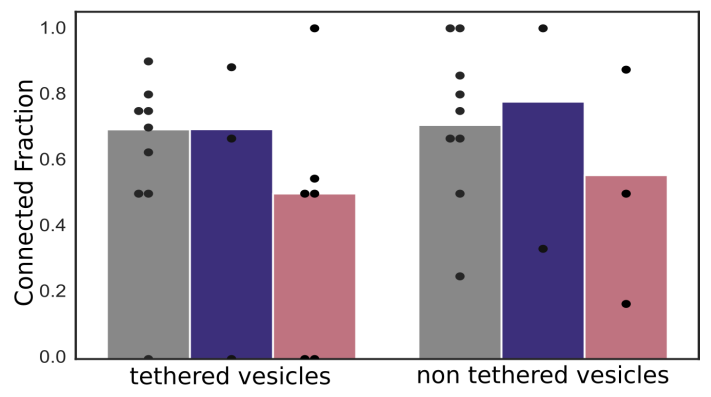

J

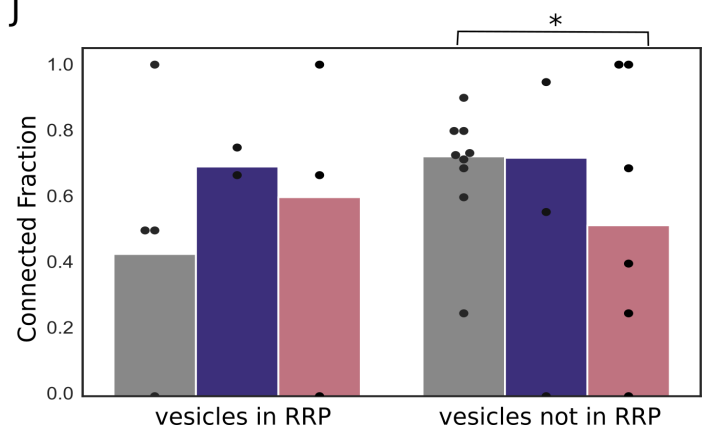

Figure S2: (A, B) Histogram of the number of tethers per proximal SV. (C, D) Histogram of the number of connectors per proximal non-RRP SV. (E, F) Histogram of the number of connectors per RRP SV. (G, H) Histogram of connected SV amongst tethered or non-tethered proximal SVs. (I, J) Histogram of connected SV amongst proximal non-RRP or RRP SVs. (A, C, E, G, I) Synapses in mouse cultured neurons. (B, D, F, H, J) Rat synaptosomes. 
bioRxiv preprint doi: https://doi.org/10.1101/2022.03.07.483217; this version posted March 7.2022. The copyright holder for this preprint (which was not certified by peer review) is the author/funder, who has granted bioRxiv a license to display the preprint in perpetuity. It is made available under aCC-BY 4.0 International license.
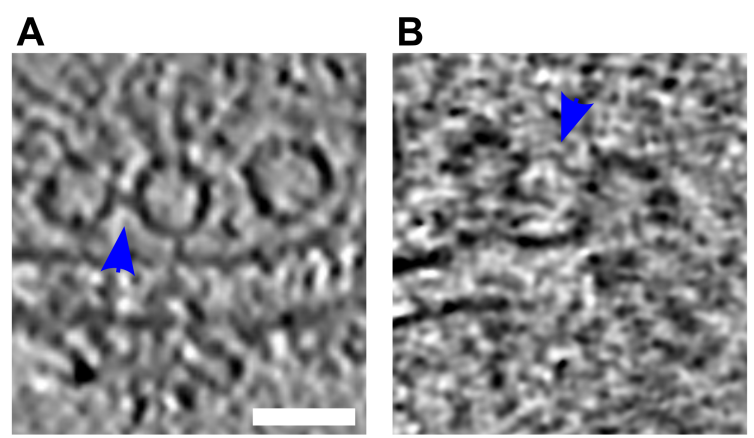

Figure S3: (A, B) Tomographic slices showing tethered connected vesicles, blue arrows highlight the connectors. Scale bar, $50 \mathrm{~nm}$

\section{Supplementary Movies}


bioRxiv preprint doi: https://doi.org/10.1101/2022.03.07.483217; this version posted March 7, 2022. The copyright holder for this preprint (which was not certified by peer review) is the author/funder, who has granted bioRxiv a license to display the preprint in perpetuity. It is made available under aCC-BY 4.0 International license.

Movie S1: Tomogram with segmentation of synaptosome with late fusion events. off-white = cell outline; pink = active zone; blue = synaptic vesicles; green = mitochondria; light green = cytomatrix; yellow $=$ connectors, red $=$ tethers, scale bar $100 \mathrm{~nm}$ 
bioRxiv preprint doi: https://doi.org/10.1101/2022.03.07.483217; this version posted March 7,2022 . The copyright holder for this preprint

(which was not certified by peer review) is the author/funder, who has granted bioRxiv a license to display the preprint in perpetuity. It is made available under aCC-BY 4.0 International license.

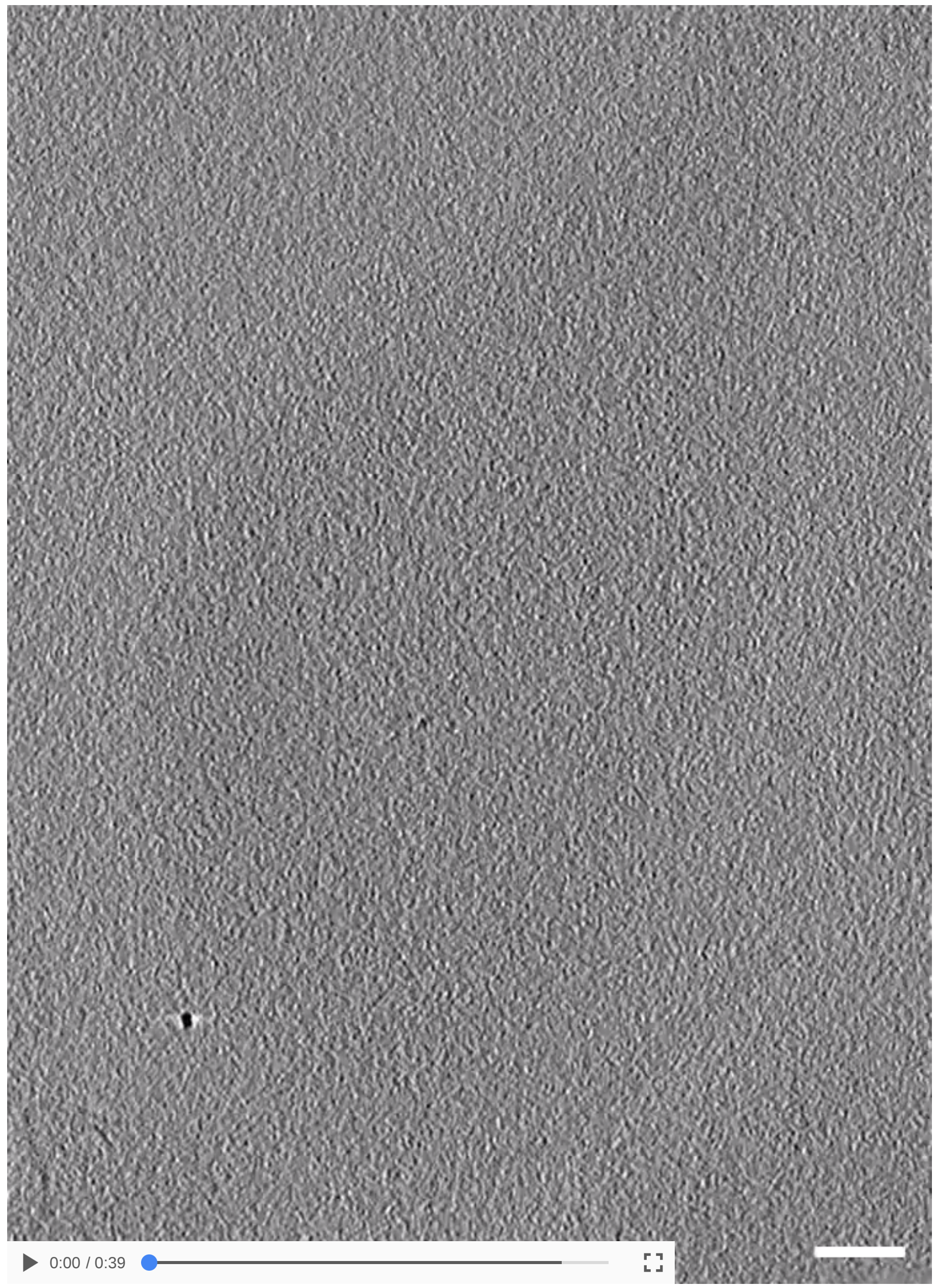

Movie S2: Tomogram with segmentation of WT SNAP-25 neurons. off-white= cell outline; pink = active zone; blue = synaptic vesicles; green = mitochondria; light green = cytomatrix; yellow = connectors, red = tethers, scale bar $100 \mathrm{~nm}$ 


\section{Author contributions}

JR, RS, JBS, and BZ designed the study. JR, RS, and Anna K performed the experiments. KG provided access to and assistance at one of the Titan Krios microscopes. JR, RS, and BZ analyzed the data. VL, UL, and Amin K contributed to the Pyto analysis. JR, RS, JBS, and BZ wrote the manuscript with contribution from all authors. JBS and BZ supervised the project.

\section{Acknowledgments}

We would like to thank Marek Kamínek for maintaining the electron microscope and supporting its use in Bern, and Tillmann Hanns Pape for support in specimen preparation and electron microscope operation in Copenhagen. Data was acquired on a machine supported by the Microscopy Imaging Center (MIC) of the University of Bern, a machine supported by the Core Facility for Integrated Microscopy_(CFIM) of the University of Copenhagen, and a machine supported by the Center for Cellular Imaging and NanoAnalytics, Biozentrum, University of Basel. This work was funded through the grants mentioned in the author list. 


\section{References}

1. Vesicle Docking in Regulated Exocytosis

Matthijs Verhage, Jakob B Sørensen

Traffic (2008-09) https://doi.org/bjtx $\underline{\text { hn }}$

DOI: $10.1111 / j .1600-0854.2008 .00759 . x \cdot P M I D: \underline{18445120}$

2. Neurotransmitter Release: The Last Millisecond in the Life of a Synaptic Vesicle

Thomas C Südhof

Neuron (2013-10) https://doi.org/f5gng $\underline{4}$

DOI: 10.1016/j.neuron.2013.10.022 · PMID: 24183019 · PMCID: PMC3866025

3. The readily releasable pool of synaptic vesicles

Pascal S Kaeser, Wade G Regehr

Current Opinion in Neurobiology (2017-04) https://doi.org/gbkfsd

DOI: $10.1016 / j . c o n b .2016 .12 .012$ P PMID: 28103533 · PMCID: PMC5447466

4. The Morphological and Molecular Nature of Synaptic Vesicle Priming at Presynaptic

Active Zones

Cordelia Imig, Sang-Won Min, Stefanie Krinner, Marife Arancillo, Christian Rosenmund, Thomas C Südhof, JeongSeop Rhee, Nils Brose, Benjamin H Cooper

Neuron (2014-10) https://doi.org/gcvj르

DOI: 10.1016/j.neuron.2014.10.009 ·PMID: 25374362

5. The Synaptic Vesicle Release Machinery

Josep Rizo, Junjie Xu

Annual Review of Biophysics (2015-06-22) https://doi.org/gjnb9q

DOI: $10.1146 /$ annurev-biophys-060414-034057 P PMID: 26098518

6. Crystal structure of a SNARE complex involved in synaptic exocytosis at $2.4 \AA$ resolution RBryan Sutton, Dirk Fasshauer, Reinhard Jahn, Axel T Brunger

Nature (1998-09) https://doi.org/cwkm8k

DOI: $\underline{10.1038 / 26412} \cdot$ PMID: $\underline{9759724}$

7. Sequential N- to C-terminal SNARE complex assembly drives priming and fusion of secretory vesicles

Jakob B Sørensen, Katrin Wiederhold, Emil M Müller, Ira Milosevic, Gábor Nagy, Bert L de Groot, Helmut Grubmüller, Dirk Fasshauer

The EMBO Journal (2006-02-23) https://doi.org/dhpwm3

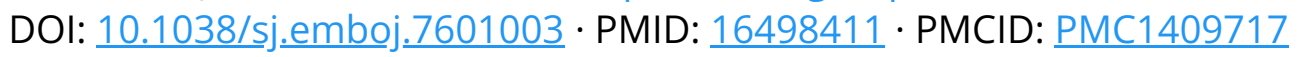

8. Single Vesicle Millisecond Fusion Kinetics Reveals Number of SNARE Complexes Optimal for Fast SNARE-mediated Membrane Fusion

Marta K Domanska, Volker Kiessling, Alexander Stein, Dirk Fasshauer, Lukas K Tamm

Journal of Biological Chemistry (2009-11) https://doi.org/chdxqp

DOI: $10.1074 / j$ bc.m109.047381 · PMID: 19759010 ·PMCID: PMC2797286

9. Fast Vesicle Fusion in Living Cells Requires at Least Three SNARE Complexes

Ralf Mohrmann, Heidi de Wit, Matthijs Verhage, Erwin Neher, Jakob B Sørensen

Science (2010-10-22) https://doi.org/c7q 87 p

DOI: $10.1126 /$ science.1193134 · PMID: 20847232

10. SNARE Proteins: One to Fuse and Three to Keep the Nascent Fusion Pore Open 
Lei Shi, Qing-Tao Shen, Alexander Kiel, Jing Wang, Hong-Wei Wang, Thomas J Melia, James E Rothman, Frédéric Pincet

Science (2012-03-16) https://doi.org/gk8gpf

DOI: 10.1126/science.1214984 · PMID: 22422984 · PMCID: PMC3736847

11. Membrane Curvature in Synaptic Vesicle Fusion and Beyond

Harvey T McMahon, Michael M Kozlov, Sascha Martens

Cell (2010-03) https://doi.org/d3gmvv

DOI: 10.1016/j.cell.2010.02.017 · PMID: 20211126

12. An Electrostatic Energy Barrier for SNARE-Dependent Spontaneous and Evoked Synaptic Transmission

Marvin Ruiter, Anna Kádková, Andrea Scheutzow, Jörg Malsam, Thomas H Söllner, Jakob B

Sørensen

Cell Reports (2019-02) https://doi.org/gfv5gd

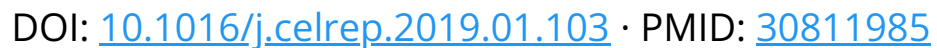

13. Quantitative analysis of the native presynaptic cytomatrix by cryoelectron tomography

Rubén Fernández-Busnadiego, Benoît Zuber, Ulrike Elisabeth Maurer, Marek Cyrklaff, Wolfgang Baumeister, Vladan Lučić

Journal of Cell Biology (2010-01-11) https://doi.org/b9c26b

DOI: 10.1083/jcb.200908082 ·PMID: 20065095 ·PMCID: PMC2812849

14. Molecular architecture of the presynaptic terminal

Benoît Zuber, Vladan Lučić

Current Opinion in Structural Biology (2019-02) https://doi.org/gk8gpd

DOI: $10.1016 / j$. sbi.2019.01.008 · PMID: $\underline{30925443}$

15. Definition of the Readily Releasable Pool of Vesicles at Hippocampal Synapses

Christian Rosenmund, Charles F Stevens

Neuron (1996-06) https://doi.org/c76twv

DOI: $\underline{10.1016 / s 0896-6273}(\underline{00}) \underline{80146-4}$

16. Properties of Synaptic Vesicle Pools in Mature Central Nerve Terminals

Anthony C Ashton, Yuri A Ushkaryov

Journal of Biological Chemistry (2005-11) https://doi.org/cb5883

DOI: $\underline{10.1074 / j \text { bc.m504137200 · PMID: } 16148008}$

17. Vesicle release probability and pre-primed pool at glutamatergic synapses in area CA1 of the rat neonatal hippocampus

Eric Hanse, Bengt Gustafsson

The Journal of Physiology (2001-03) https://doi.org/fq7th3

DOI: 10.1111/j.1469-7793.2001.0481i.X ·PMID: 11230520 ·PMCID: PMC2278469

18. Reluctant Vesicles Contribute to the Total Readily Releasable Pool in Glutamatergic Hippocampal Neurons

KL Moulder

Journal of Neuroscience (2005-04-13) https://doi.org/dwqpxj

DOI: 10.1523/jneurosci.5231-04.2005 · PMID: 15829636 · PMCID: PMC6724923

19. Synaptic vesicle pools: an update

Denker

Frontiers in Synaptic Neuroscience (2010) https://doi.org/d74dd7

DOI: 10.3389/fnsyn.2010.00135 · PMID: 21423521 · PMCID: PMC3059705 
20. The cytoskeletal architecture of the presynaptic terminal and molecular structure of synapsin 1.

N Hirokawa, K Sobue, K Kanda, A Harada, H Yorifuji

Journal of Cell Biology (1989-01-01) https://doi.org/ddbqhb

DOI: 10.1083/jcb.108.1.111 · PMID: 2536030 · PMCID: PMC2115350

21. Three-Dimensional Architecture of Presynaptic Terminal Cytomatrix

L Siksou, P Rostaing, J-P Lechaire, T Boudier, T Ohtsuka, A Fejtova, H-T Kao, P Greengard, ED Gundelfinger, A Triller, S Marty

Journal of Neuroscience (2007-06-27) https://doi.org/bjw3mv

DOI: 10.1523/jneurosci.1773-07.2007 · PMID: 17596435 · PMCID: PMC6672225

22. Physical determinants of vesicle mobility and supply at a central synapse

Jason Seth Rothman, Laszlo Kocsis, Etienne Herzog, Zoltan Nusser, Robin Angus Silver

eLife (2016-08-19) https://doi.org/f9rmkt

DOI: 10.7554/elife.15133 · PMID: 27542193 · PMCID: PMC5025287

23. Activity-Dependence of Synaptic Vesicle Dynamics

Luca A Forte, Michael W Gramlich, Vitaly A Klyachko

The Journal of Neuroscience (2017-09-27) https://doi.org/gch7j 3

DOI: 10.1523/jneurosci.0383-17.2017 · PMID: 28954868 · PMCID: PMC5666583

24. Synapsin dispersion and reclustering during synaptic activity

Ping Chi, Paul Greengard, Timothy A Ryan

Nature Neuroscience (2001-10-29) https://doi.org/cpdwc7

DOI: $10.1038 / \mathrm{nn} 756$ ·PMID: 11685225

25. Interactions of synapsin I with small synaptic vesicles: distinct sites in synapsin I bind to vesicle phospholipids and vesicle proteins.

F Benfenati, M Bähler, R Jahn, P Greengard

Journal of Cell Biology (1989-05-01) https://doi.org/d65t4v

DOI: 10.1083/jcb.108.5.1863 ·PMID: 2497106 · PMCID: PMC2115532

26. Visualization of Synaptic Vesicle Movement in Intact Synaptic Boutons Using

Fluorescence Fluctuation Spectroscopy

Randolf Jordan, Edward A Lemke, Jurgen Klingauf

Biophysical Journal (2005-09) https://doi.org/cdpkkv

DOI: 10.1529/biophysj.105.061663 · PMID: 15980175 ·PMCID: PMC1366711

27. Synapsin Selectively Controls the Mobility of Resting Pool Vesicles at Hippocampal Terminals

A Orenbuch, L Shalev, V Marra, I Sinai, Y Lavy, J Kahn, JJ Burden, K Staras, D Gitler

Journal of Neuroscience (2012-03-21) https://doi.org/£3wq83

DOI: 10.1523/jneurosci.5058-11.2012 · PMID: 22442064 · PMCID: PMC3492757

28. High Mobility of Vesicles Supports Continuous Exocytosis at a Ribbon Synapse

Matthew Holt, Anne Cooke, Andreas Neef, Leon Lagnado

Current Biology (2004-02) https://doi.org/b9g9h2

DOI: $\underline{10.1016 / j}$.cub.2003.12.053 ·PMID: 14761649

29. Correlative microscopy: Bridging the gap between fluorescence light microscopy and cryo-electron tomography

Anna Sartori, Rudolf Gatz, Florian Beck, Alexander Rigort, Wolfgang Baumeister, Juergen M Plitzko

Journal of Structural Biology (2007-11) https://doi.org/cp $5 \mathrm{krz}$ 
DOI: $10.1016 / j . j s$ b. 2007.07.011 · PMID: 17884579

30. Analysis of transient structures by cryo-microscopy combined with rapid mixing of spray droplets

John Berriman, Nigel Unwin

Ultramicroscopy (1994-12) https://doi.org/ctwp5j

DOI: 10.1016/0304-3991(94) $\underline{90012-4}$

31. Cryo-electron tomography reveals a critical role of RIM1a in synaptic vesicle tethering Rubén Fernández-Busnadiego, Shoh Asano, Ana-Maria Oprisoreanu, Eri Sakata, Michael Doengi, Zdravko Kochovski, Magdalena Zürner, Valentin Stein, Susanne Schoch, Wolfgang Baumeister, Vladan Lučić Journal of Cell Biology (2013-05-27) https://doi.org/f4x2mj DOI: 10.1083/jcb.201206063 ·PMID: $23712261 \cdot P M C I D: ~ P M C 3664715$

32. SNARE and regulatory proteins induce local membrane protrusions to prime docked vesicles for fast calcium-triggered fusion

Tanmay AM Bharat, Jörg Malsam, Wim JH Hagen, Andrea Scheutzow, Thomas H Söllner, John AG Briggs

EMBO reports (2014-02-03) https://doi.org/f2qg $\underline{4 d}$

DOI: 10.1002/embr.201337807 ·PMID: 24493260 · PMCID: PMC3989697

33. Phosphatidylinositol 4,5 Bisphosphate Controls the cis and trans Interactions of Synaptotagmin 1

Sarah B Nyenhuis, Anusa Thapa, David S Cafiso

Biophysical Journal (2019-07) https://doi.org/gnk9cp

DOI: 10.1016/j.bpj.2019.06.016 · PMID: $\underline{31301806}$ · PMCID: PMC6701194

34. Single Reconstituted Neuronal SNARE Complexes Zipper in Three Distinct Stages

Ying Gao, Sylvain Zorman, Gregory Gundersen, Zhiqun Xi, Lu Ma, George Sirinakis, James E Rothman, Yongli Zhang

Science (2012-09-14) https://doi.org/f37csk

DOI: 10.1126/science.1224492 · PMID: 22903523 ·PMCID: PMC3677750

35. Molecular mechanism of fusion pore formation driven by the neuronal SNARE complex Satyan Sharma, Manfred Lindau

Proceedings of the National Academy of Sciences (2018-11-27) https://doi.org/gfsd3m

DOI: 10.1073/pnas.1816495115 · PMID: $\underline{30482862}$ · PMCID: PMC6294955

36. Caught in the Act: Visualization of SNARE-Mediated Fusion Events in Molecular Detail Herre Jelger Risselada, Carsten Kutzner, Helmut Grubmüller

ChemBioChem (2011-03-23) https://doi.org/bwr34v

DOI: $10.1002 /$ cbic.201100020 · PMID: 21433241

37. Synaptotagmin-1 may be a distance regulator acting upstream of SNARE nucleation Geert van den Bogaart, Shashi Thutupalli, Jelger H Risselada, Karsten Meyenberg, Matthew Holt, Dietmar Riedel, UIf Diederichsen, Stephan Herminghaus, Helmut Grubmüller, Reinhard Jahn Nature Structural \& Molecular Biology (2011-06-05) https://doi.org/dh26xp DOI: 10.1038/nsmb.2061 · PMID: 21642968 · PMCID: PMC3130798

38. Synaptobrevin N-terminally bound to syntaxin-SNAP-25 defines the primed vesicle state in regulated exocytosis

Alexander M Walter, Katrin Wiederhold, Dieter Bruns, Dirk Fasshauer, Jakob B Sørensen Journal of Cell Biology (2010-02-08) https://doi.org/bsp5dq

DOI: 10.1083/jcb.200907018 · PMID: 20142423 · PMCID: PMC2819690 
bioRxiv preprint doi: https://doi.org/10.1101/2022.03.07.483217; this version posted March $7,2022$. The copyright holder for this preprint (which was not certified by peer review) is the author/funder, who has granted bioRxiv a license to display the preprint in perpetuity. It is made available under aCC-BY 4.0 International license.

39. Opposing functions of two sub-domains of the SNARE-complex in neurotransmission Jens P Weber, Kerstin Reim, Jakob B Sørensen

The EMBO Journal(2010-06-18) https://doi.org/dzkrh2

DOI: 10.1038/emboj.2010.130 · PMID: 20562829 · PMCID: PMC2928689

40. Dynamics and number of trans-SNARE complexes determine nascent fusion pore properties

Huan Bao, Debasis Das, Nicholas A Courtney, Yihao Jiang, Joseph S Briguglio, Xiaochu Lou, Daniel Roston, Qiang Cui, Baron Chanda, Edwin R Chapman

Nature (2018-01-31) https://doi.org/gczkdz

DOI: 10.1038/nature25481 · PMID: 29420480 ·PMCID: PMC5808578

41. Superpriming of synaptic vesicles as a common basis for intersynapse variability and modulation of synaptic strength

Holger Taschenberger, Andrew Woehler, Erwin Neher

Proceedings of the National Academy of Sciences (2016-07-18) https://doi.org/f8w9vc

DOI: 10.1073/pnas.1606383113 · PMID: 27432975 · PMCID: PMC4978258

42. Superpriming of synaptic vesicles after their recruitment to the readily releasable pool Jae Sung Lee, Won-Kyung Ho, Erwin Neher, Suk-Ho Lee

Proceedings of the National Academy of Sciences (2013-08-26) https://doi.org/1/494g 4

DOI: 10.1073/pnas.1314427110 · PMID: 23980146 · PMCID: PMC3773744

43. Merits and Limitations of Vesicle Pool Models in View of Heterogeneous Populations of Synaptic Vesicles

Erwin Neher

Neuron (2015-09) https://doi.org/f7whxq

DOI: 10.1016/j.neuron.2015.08.038 · PMID: 26402599

44. Rapid regulation of vesicle priming explains synaptic facilitation despite heterogeneous vesicle:Ca2+ channel distances

Janus RL Kobbersmed, Andreas T Grasskamp, Meida Jusyte, Mathias A Böhme, Susanne

Ditlevsen, Jakob Balslev Sørensen, Alexander M Walter

eLife (2020-02-20) https://doi.org/gnf2f5

DOI: 10.7554/elife.51032 · PMID: 32077852 P PMCID: PMC7145420

45. Control of Presynaptic Parallel Fiber Efficacy by Activity-Dependent Regulation of the Number of Occupied Release Sites

Hartmut Schmidt

Frontiers in Systems Neuroscience (2019-07-17) https://doi.org/gnk9cw

DOI: 10.3389/fnsys.2019.00030 · PMID: 31379524 · PMCID: PMC6650762

46. Calcium-dependent docking of synaptic vesicles

Melissa Silva, Van Tran, Alain Marty

Trends in Neurosciences (2021-07) https://doi.org/gkzxh9

DOI: 10.1016/j.tins.2021.04.003 · PMID: 34049722

47. Synaptic vesicles transiently dock to refill release sites

Grant F Kusick, Morven Chin, Sumana Raychaudhuri, Kristina Lippmann, Kadidia P Adula, Edward J Hujber, Thien Vu, MWayne Davis, Erik M Jorgensen, Shigeki Watanabe

Nature Neuroscience (2020-09-28) https://doi.org/gmghk9

DOI: 10.1038/s41593-020-00716-1 ·PMID: 32989294 ·PMCID: PMC8054220

48. Actin- and Myosin-Dependent Vesicle Loading of Presynaptic Docking Sites Prior to Exocytosis 
bioRxiv preprint doi: https://doi.org/10.1101/2022.03.07.483217; this version posted March 7,2022 . The copyright holder for this preprint (which was not certified by peer review) is the author/funder, who has granted bioRxiv a license to display the preprint in perpetuity. It is made available under aCC-BY 4.0 International license.

Takafumi Miki, Gerardo Malagon, Camila Pulido, Isabel Llano, Erwin Neher, Alain Marty Neuron (2016-08) https://doi.org/f8z4sn DOI: 10.1016/j.neuron.2016.07.033 · PMID: 27537485

49. Differential Abilities of SNAP-25 Homologs to Support Neuronal Function I Delgado-Martinez, RB Nehring, JB Sorensen Journal of Neuroscience (2007-08-29) https://doi.org/d7mhxn DOI: 10.1523/jneurosci.5092-06.2007 · PMID: 17728451 · PMCID: PMC6673127

50. A rapid Percoll gradient procedure for preparation of synaptosomes

Peter R Dunkley, Paula E Jarvie, Phillip J Robinson

Nature Protocols (2008-10-16) https://doi.org/b7zwh8

DOI: $10.1038 /$ nprot.2008.171 · PMID: 18927557

51. Preparation of Primary Neurons for Visualizing Neurites in a Frozen-hydrated State Using Cryo-Electron Tomography

Sarah H Shahmoradian, Mauricio R Galiano, Chengbiao Wu, Shurui Chen, Matthew N Rasband, William C Mobley, Wah Chiu Journal of Visualized Experiments (2014-02-12) https://doi.org/gmh9w3 DOI: $10.3791 / 50783$ · PMID: 24561719 · PMCID: PMC4089403

52. Genetic ablation of the t-SNARE SNAP-25 distinguishes mechanisms of neuroexocytosis Philip Washbourne, Peter M Thompson, Mario Carta, Edmar T Costa, James R Mathews, Guillermina Lopez-Benditó, Zoltán Molnár, Mark W Becher, CFernando Valenzuela, LDonald Partridge, Michael C Wilson

Nature Neuroscience (2001-12-19) https://doi.org/fbmxgc

DOI: $10.1038 / \mathrm{nn} 783 \cdot$ PMID: 11753414

53. Automated electron microscope tomography using robust prediction of specimen movements

David N Mastronarde

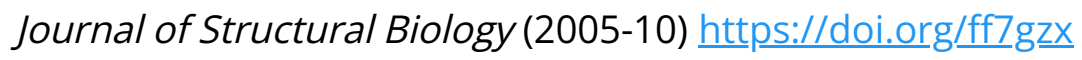

DOI: 10.1016/j.js.b.2005.07.007 • PMID: 16182563

54. 2dx_automator: Implementation of a semiautomatic high-throughput high-resolution cryo-electron crystallography pipeline

Sebastian Scherer, Julia Kowal, Mohamed Chami, Venkata Dandey, Marcel Arheit, Philippe Ringler, Henning Stahlberg

Journal of Structural Biology (2014-05) https://doi.org/f522h3

DOI: 10.1016/j.js.j. 2014.03.016 · PMID: 24680783

55. Electron counting and beam-induced motion correction enable near-atomic-resolution single-particle cryo-EM

Xueming Li, Paul Mooney, Shawn Zheng, Christopher R Booth, Michael B Braunfeld, Sander Gubbens, David A Agard, Yifan Cheng Nature Methods (2013-05-05) https://doi.org/f4zpj $\underline{f}$

DOI: 10.1038/nmeth.2472 · PMID: 23644547 ·PMCID: PMC3684049

56. Computer Visualization of Three-Dimensional Image Data Using IMOD

James R Kremer, David N Mastronarde, JRichard McIntosh

Journal of Structural Biology (1996-01) https://doi.org/d9nfzw

DOI: 10.1006/jsbi.1996.0013 · PMID: $\underline{8742726}$

57. A rapid Percoll gradient procedure for isolation of synaptosomes directly from an S1 fraction: viability of subcellular fractions 
bioRxiv preprint doi: https://doi.org/10.1101/2022.03.07.483217; this version posted March 7, 2022. The copyright holder for this preprint (which was not certified by peer review) is the author/funder, who has granted bioRxiv a license to display the preprint in perpetuity. It is made available under aCC-BY 4.0 International license.

Steven M Harrison, Paula E Jarvie, Peter R Dunkley

Brain Research (1988-02) https://doi.org/b5tzcr

DOI: $10.1016 / 0006-8993(\underline{88}) \underline{91384-4}$

58. Hierarchical detection and analysis of macromolecular complexes in cryo-electron tomograms using Pyto software

Vladan Lučić, Rubén Fernández-Busnadiego, Ulrike Laugks, Wolfgang Baumeister Journal of Structural Biology (2016-12) https://doi.org/f9d5t2

DOI: 10.1016/j:jsb.2016.10.004 · PMID: 27742578

59. Open collaborative writing with Manubot

Daniel S Himmelstein, Vincent Rubinetti, David R Slochower, Dongbo Hu, Venkat S Malladi, Casey S Greene, Anthony Gitter

PLOS Computational Biology (2019-06-24) https://doi.org/c7np

DOI: 10.1371/journal.pcbi.1007128 · PMID: 31233491 · PMCID: PMC6611653 